\title{
Damage identification in bars with a wave propagation approach and a hybrid optimization method
}

\author{
R.A. Tenenbaum*, K.M. Fernandes, L.T. Stutz and A.J. Silva Neto \\ Graduate Program in Computational Modeling, Polytechnic Institute, State University of Rio de Janeiro, Rua \\ Alberto Rangel S/N, Vila Nova, Nova Friburgo, RJ, Brazil
}

Received 1 April 2010

Revised 16 December 2010

\begin{abstract}
The formulation and solution of the inverse problem of damage identification based on wave propagation approach are presented. Different damage scenarios for a bar are considered. Time history responses, obtained from pulse-echo synthetic experiments, are used to identify damage position, severity and shape. In order to account for noise corrupted data, different levels of signal to noise ratio - varying from 30 to $0 \mathrm{~dB}$ - are introduced. In the identification process, different optimization methods are investigated: the deterministic Levenberg-Marquardt; the stochastic Particle Swarm Optimization; and a hybrid technique combining the aforementioned methods. It is shown that the damage identification procedure built on the wave propagation approach was successful, even for highly corrupted noisy data. Test case results are presented and a few comments on the advantages of deterministic and stochastic methods and their combination are also reported. Finally, an experimental validation of the sequential algebraic algorithm, used for modeling the direct problem, is presented.
\end{abstract}

Keywords: Structural damage identification, wave propagation in solids, particle swarm optimization, hybrid optimization method

\section{Introduction}

Damage identification can be addressed from the point of view of wave propagation approach. It is well known that damage identification approaches built on wave propagation are highly sensitive to changes in local dynamic impedance [8-10], such as those caused by small defects. Although these approaches are much more uncommon in the literature than the vibration ones [17,33,43], they have the advantage of being directly defined in the time domain, yielding fast techniques with good accuracy [39]. Wave propagation has been considered as a useful model for damage assessment of structural elements, using Lamb waves [30,44]. More recently, Manson and Barthorpe [20] presented a new approach for damage location using a statistically calculated threshold.

Applications of wave propagation in inverse problems are classically reported in the fields of geophysics [22,32], medical ultrasonics [18], fatigue testing [7,25], non-destructive testing [37], and, more recently, in the evaluation of the integrity of drilled piles [26-28]. The wave propagation approach has also been applied to damage assessment in composite materials [19] and in the identification of delamination [24]. It is also found in the literature some works dealing with two-dimensional wave propagation, as in [16] and in [41]. Two very comprehensive surveys on this subject are [1,23].

The main goal of this research is to study the inverse problem of damage identification in a bar within the framework of wave propagation approach. In this same subject some papers can be found in the literature, where

\footnotetext{
*Corresponding author. Tel.: +55 22 25288300; Fax: +55 22 25332322; E-mail: ratenenbaum@ gmail.com.
} 
the identification problem is solved, for instance, using genetic algorithms [24], global methods [34] and even a sequential method [38]. However, none of the works found in the literature [1,23] deals with a sequential algebraic algorithm that yields a very fast and accurate solution for the direct problem and, besides, enables the identification of a relatively large number of parameters, which provides a technique that is robust to noise corrupted signals. This, together with the use of a hybrid technique in the identification procedure, which will be shown to be computationally efficient, is the most important contribution of the present work to the field.

In the present work, the direct problem of one-dimensional wave propagation is first addressed by considering the Sequential Algebraic Algorithm (SAA) presented in [38]. In the sequel, the inverse problem of damage identification is addressed, in the time domain, by minimizing the difference between the experimental (or synthetic) echo and the one predicted by the SAA. Three optimization methods were considered in the identification process. The first one is the classical deterministic method of Levenberg-Marquardt (LM) [21]. The second one is the stochastic Particle Swarm Optimization (PSO) method [15,35]. Finally, the third technique (PSO-LM) is a hybrid one, where, essentially, the PSO method is used to generate an initial guess for the LM one. An experimental validation of the above methods is also presented.

The main interest in using a hybrid method for this purpose relies on the fact that the results provided by the LM technique, as will be shown in Section 4, depend strongly on an arbitrary parameter (relaxation factor). For an unsuitable choice of this parameter the result of the LM method may even diverge. On the other hand, due to its stochastic nature, the PSO method results usually yield to a relatively large residual error in the identification. The combination of the two techniques, however, provides the best damage assessment results.

For each damage scenario, a pulse-echo synthetic experiment is performed and the excitation and corresponding response are considered in the damage identification procedure. In the present work, the excitation is considered as a longitudinal impact at one end of a bar with length $l$, which generates progressive (pulse) and regressive (echo) strain waves propagating along it. Firstly, the excitation pulses considered in this work are of the kind of Dirac's delta, $\delta(t)$. In the sequel, rectangular pulses of two kinds (short and long pulses) are considered as excitation functions. As it will be shown, there are no noticeable differences in the identification results, for the three excitation signals.

Since the echo is observed in a restricted time interval $\Delta T=2 l_{u} / c$, where $l_{u}$ is the distance of the strain sensor to the other end of the bar and $c$ is the longitudinal wave speed, the expected undamaged bar response is null. On the other hand, in a damaged bar, the inhomogeneity due to the damage generates a non-null echo within the considered time interval $\Delta T$.

In this work, the damage is assumed to correspond to a function $A(x)$, were $A$ is the cross section area in a certain (and unknown) spatial domain $x \in\left(x_{1}, x_{2}\right)$, which generates a non-null echo. Therefore, the identification is performed minimizing, with respect to the cross-section area, the squared norm between the true echo and the predicted one.

The paper is organized as follows. In Section 2 a summary of the theoretical wave propagation model is presented, together with a description of the inhomogeneity scenarios addressed. In Section 3 the adopted optimization procedures are displayed. The results for the damage identification with noiseless data are shown in Section 4, which includes an analysis of the effects of the relaxation factor $\Gamma$ on the solution with the LM method. In Section 5 the results for the damage assessment with noisy data for different damage scenarios are presented and discussed. An actual experimental setup, aiming at validating the proposed damage identification method, is described in Section 6. The general conclusions are exhibited in Section 7.

\section{Problem formulation}

\subsection{Mathematical model for the direct problem}

The one-dimensional longitudinal wave propagation in a non-homogeneous slender bar can be described by the hyperbolic second-order differential equation

$$
\sigma_{t t}-c^{2}\left[\sigma_{x x}+\left(\frac{A^{\prime}}{A}-\frac{\rho^{\prime}}{\rho}\right) \sigma_{x}+\rho\left(\frac{A^{\prime}}{\rho A}\right)^{\prime} \sigma\right]=0,
$$


where $\sigma(x, t)$ is the longitudinal stress field, depending on the position $x$ and time $t, A(x)$ is the bar cross section area, $\rho(x)$ is the bar density, both depending on the position $x, c$ is the longitudinal wave velocity, the prime stands for the total derivative, and the subscripts, as usual, represent partial derivatives.

The general D'Alembert solution for Eq. (1) cannot be obtained in a closed form. However, it can be shown [38], that Eq. (1) can be written in an alternative form, in the characteristic plane $(r, s)$, as the following system of first-order equations:

$$
\begin{aligned}
& U_{r}+\frac{\dot{Z}}{4 Z} U=0 ; \\
& V_{s}-\frac{\dot{Z}}{4 Z} V=0,
\end{aligned}
$$

where $Z=\rho c A$ is the generalized acoustical impedance, $U(r, s)$ and $V(r, s)$ are, respectively, the progressive and regressive stress wave components traveling along the characteristic plane, defined as

$$
\begin{aligned}
& r=t+\tau ; \\
& s=t-\tau,
\end{aligned}
$$

and the dot stands as derivative with respect to the independent variable $\tau$, the travel time, defined as

$$
\tau(x)=\int_{0}^{x} \frac{d \xi}{c(\xi)}
$$

Equation (2) is a compact and uncoupled pair of first order differential equations that describes the longitudinal wave propagation phenomenon in a more convenient way. To integrate it, boundary conditions in the $(r, s)$ plane must be provided, corresponding to the physical situation under concern. Let us, for instance, consider the probing of a medium, $x \geqslant 0$, by a pulse excitation at $x=0$. Assuming also the Sommerfeld radiation hypothesis [29], the boundary conditions can be stated as:

$$
U(s, s)=F(s)=f(t) ; \quad V(r, 0)=0,
$$

where $f(t)$ is the incident longitudinal stress being applied at the boundary $r=s(x=0)$ and the second equation ensures that there is no disturbance in $s \leqslant 0(t \leqslant x / c)$, where $c$ is the wave speed. Note that $f(t)$, being the longitudinal stress at the physical boundary $x=0$, corresponds to $U(s, s)$, a progressive wave component. Analogously, the echo observed at $x=0$, due to the damage, will be the output signal $g(t)=V(s, s)$, a regressive wave component.

Assuming now the bar under study as a sectionally homogeneous discretized one with equal length $\Delta x=c \Delta t$, the known discrete incoming pulse is written as

$$
F_{j}=f(2(j-1) \Delta t)
$$

and the discrete outgoing echo will be

$$
G_{j}=g(2 j \Delta t), \quad j=1,2, \ldots, N,
$$

where $N \Delta t$ is the total time interval under consideration.

It can be shown that Eq. (2), with the boundary conditions given in Eq. (5), have, after the discretization given in Eqs (6) and (7), the following algebraic solution for the echo [38]

$$
G_{j}=\sum_{k=1}^{j}\left(R_{k}+\sum_{p=1}^{k-2} Q_{k}^{p}\right) F_{j-k+1},
$$

where the polynomials $Q_{k}^{p}$ have the general recursive formula

$$
\begin{array}{r}
Q_{k}^{p}=R_{k-p}\left[\frac{Q_{k-1}^{p}}{R_{k-p-1}}-R_{k-p-1}\left(R_{k-1}+\sum_{l=1}^{p-1} Q_{k-1}^{l}\right)\right], \quad k=1,2, \ldots, N \\
p=1,2, \ldots, k-2 .
\end{array}
$$




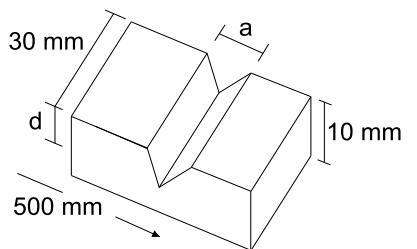

(a) Case 1

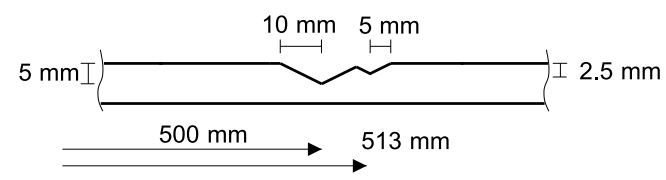

(c) Case 3

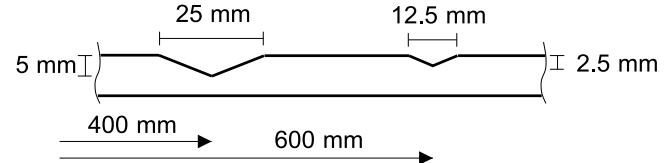

(b) Case 2

Fig. 1. Four different damage scenarios imposed to the slender bar.

In Eq. (9), $R_{i}$ stands for the reflection coefficient at the $i$-th layer of the medium, defined as

$$
R_{i}=\frac{Z_{i}-Z_{i-1}}{Z_{i}+Z_{i-1}}, \quad i=1,2, \ldots, n,
$$

where $Z_{i}=\rho c A_{i}$ is the generalized acoustical impedance of the medium [38]. Since $\rho$ and $c$ are assumed as constant, the reflection coefficient can be written as

$$
R_{i}=\frac{A_{i}-A_{i-1}}{A_{i}+A_{i-1}}, \quad i=1,2, \ldots, n .
$$

The mathematical procedure, in the direct wave propagation approach, consists then in the following steps. The medium, with the nominal cross-section area $A_{0}$ and the nominal generalized acoustical impedance $Z_{0}$, is discretized into $n$ elements. Then, the reflection coefficients are computed by Eq. (11). In the sequel, the polynomials $Q_{k}^{p}$ are calculated from Eq. (9). Finally, the output echo is computed from Eq. (8).

It is worth stressing that the mathematical model above provides an original algebraic formula to solve the direct wave propagation problem. It also permits, in the identification procedure, to identify one parameter per step. As it will be seen in the damage assessment results, the number of parameters that may be updated in the identification is significantly larger than what is usual in optimization processes [4,31].

It is worth noting that it is not necessary to consider an infinite or even a semi-infinite medium. The echoes will be observed in the interval $t \in\left(0,2 l_{u} / c\right)$, where $l_{u}$ is the distance from the strain gage station to the end of the bar. This means that the echo originated in the other bar end - whatever is its boundary condition - is irrelevant.

\subsection{Damage scenarios addressed}

The structure under consideration is a aluminium bar with 1 meter in length and with a nominal rectangular cross-section of $30 \mathrm{~mm} \times 10 \mathrm{~mm}$, see Fig. 1 . The material has elastic modulus $E_{a}=7.1 \times 10^{10} \mathrm{~Pa}$, density $\rho=2.7 \times 10^{3} \mathrm{~kg} / \mathrm{m}^{3}$ and longitudinal plane wave speed $c=5128 \mathrm{~m} / \mathrm{s}$. The strain gage sensor is considered at $10 \mathrm{~mm}$ far from the impact bar end. As it is well known, such slender bar works as a plane waveguide, as demonstrated by experimental tests in [39] and [27], and the one-dimensional model applies quite well. In Section 6, an experimental validation of the proposed model is also presented.

Four different kinds of damage, referred to as Case 1 to 4 , were imposed to the bar, as depicted in Fig. 1. The corresponding impulse responses for the four damage cases are presented in Fig. 2. These signals were obtained with the spatial discretization $\Delta x=1 \mathrm{~mm}$. For obtaining the impulse response of Case 1, damage parameters $a$ and $d$ was adopted was $25 \mathrm{~mm}$ and $5 \mathrm{~mm}$, respectively. 


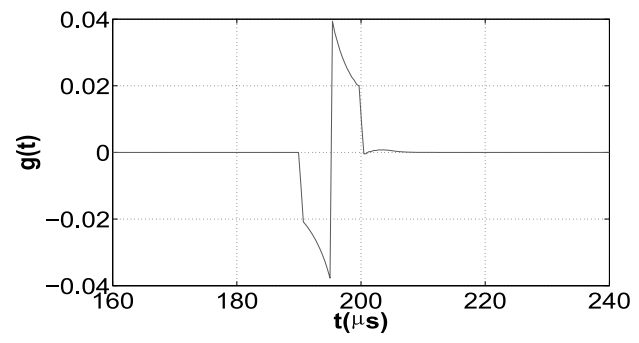

(a) Case 1

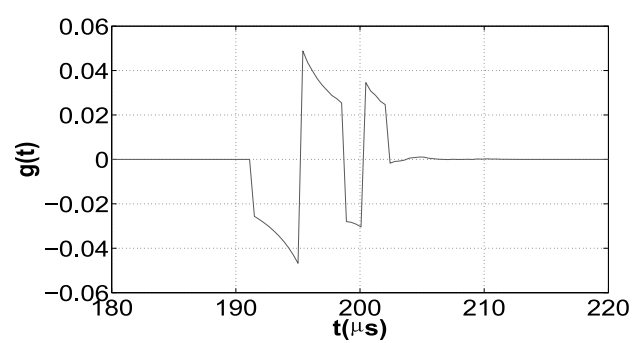

(c) Case 3

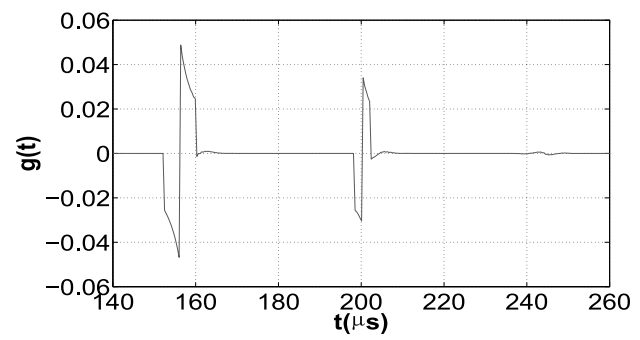

(b) Case 2

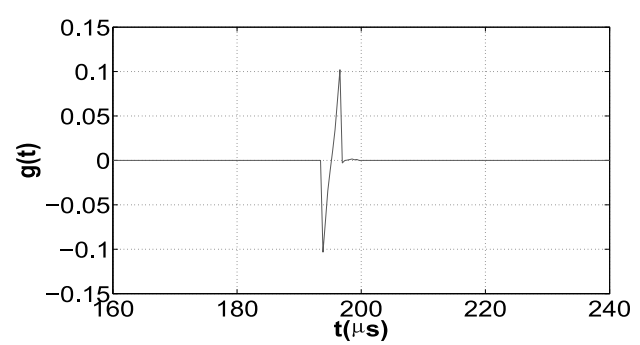

(d) Case 4

Fig. 2. Impulse responses from the pulse-echo tests for the four damage cases.

These are the functions that, when discretized, will provide the set of data for the identification process. The echoes presented in Fig. 2 were obtained running the algebraic algorithm given by Eqs (7), (9) and (11), for an impulsive excitation.

It is worth noting that the echoes themselves provide several and important information about the damage. For instance, the echo depicted in Fig. 2a, begins at $190 \mu$ s and ends at about $200 \mu$ s. This means that, considering the plane wave speed $c$ in the present case, the damage must begin at $487.5 \mathrm{~mm}$ and end at $512.5 \mathrm{~mm}$, which corresponds to the damage depicted in Fig. 1a. Of course, the damage shape cannot be identified without solving the corresponding inverse problem.

\section{Identification procedures}

For damage identification purposes, the bar is spatially discretized into $n$ sections with equal length, so that the area profile $A(x)$ is approximated by sectionally constant values $A_{i}, i=1,2, \ldots n$.

Defining the vector

$$
\mathbf{A}=\left\{A_{1}, A_{2}, \ldots, A_{n}\right\},
$$

the damage identification problem under consideration may be posed as a finite dimensional minimization one as follows.

$$
\min _{\mathbf{A}} E
$$

where the functional $E$ is the squared norm of the residue vector $\mathbf{r}(\mathbf{A})$, which is defined as

$$
\mathbf{r}(\mathbf{A})=\left\{\begin{array}{c}
G_{1}(\mathbf{A})-\bar{G}_{1} \\
G_{2}(\mathbf{A})-\bar{G}_{2} \\
\vdots \\
G_{N}(\mathbf{A})-\bar{G}_{N}
\end{array}\right\} .
$$

In Eq. (14), $N$ is the number of data considered in the identification process, $G_{j}(\mathbf{A})$ is the echo predicted by the model, and $\bar{G}_{j}$ is the synthetic echo at the time instant $t_{j}$. Therefore, from Eq. (14), one has 


$$
E=\mathbf{r}^{T} \mathbf{r}=\sum_{j=1}^{N}\left[G_{j}(\mathbf{A})-\bar{G}_{j}\right]^{2}
$$

where the superscript $T$ denotes transpose.

In this work, aiming at solving the identification problem in (13), the Levenberg-Marquardt method, the Particle Swarm Optimization method, and a hybrid method, combing the aforementioned ones, are considered.

It is important to emphasize that the previous analysis of the echo provides useful information for the identification procedure. For instance, taking the echo depicted in Fig. 2a, it was possible to infer about the damage location (from 487.5 to $512.5 \mathrm{~mm}$, as seen in Section 2.2) and, therefore, only the parameters $A_{i}$ corresponding to this region needs to be updated in the identification process.

\subsection{The Levenberg-Marquardt method}

Briefly, the deterministic Levenberg-Marquardt (LM) method [21] consists in constructing an iterative procedure, which starts with an initial guess $\mathbf{A}^{0}$, and, at the $(k+1)$-th iteration, the new estimate is given by

$$
\mathbf{A}^{k+1}=\mathbf{A}^{k}+\Delta \mathbf{A}^{k}, \quad k=0,1, \ldots,
$$

with the variation $\Delta \mathbf{A}^{k}$ being computed from

$$
\Delta \mathbf{A}^{k}=-\left(\left(\mathbf{J}^{T}\right)^{k} \mathbf{J}^{k}+\lambda^{k} \mathbf{I}\right)^{-1}\left(\mathbf{J}^{T}\right)^{k} \Gamma \mathbf{r}^{k},
$$

where $\lambda$ is a damping parameter that is adjusted at each iteration, $\mathbf{I}$ is the identity matrix, $\Gamma$ is a relaxation factor, and the elements of the Jacobian matrix $\mathbf{J}$ are defined as

$$
J_{j i}=\frac{\partial G_{j}}{\partial A_{i}}, \quad j=1,2, \ldots, N, \quad i=1,2, \ldots, n .
$$

The iterative procedure is continued until the convergence criteria, $\left|E^{k}\right|<\epsilon_{1}$ or $\left|E^{k+1}-E^{k}\right|<\epsilon_{2}$, are satisfied, where $\epsilon_{1}$ and $\epsilon_{2}$ are arbitrarily small numbers.

\subsection{The particle swarm optimization method}

The PSO is a population based search algorithm. It was inspired from natural behavior of animals [12-14]. The population contains a set of individuals, or agents, referred to as particles, where each one represents a possible solution for a given optimization problem. These particles are, in general, randomly initialized. During the PSO process, each particle, based on a given evaluation criterion, updates its own position with a certain speed, which is computed based on both the best experience of the particle itself and that of the entire population. This update process is repeated for a number of generations. The update process stops either when the objective is achieved or when the maximum number of generations is reached. PSO based techniques were applied to very distinct applications, as in telecommunications [6], robotics [2], to extract rules from a fuzzy neural network [11] and in cooperatively learning based neural network [5].

The PSO algorithm can be described in four steps: 1. initial generation of each particle state (position and velocity); 2. update of each particle velocity; 3 . update of each particle position; and 4 . check of stopping criteria.

Consider a swarm with $m$ particles. The positions $x_{i, k}$ and velocities $v_{i, k}$ of the initial swarm of particles will be initialized randomly within a specific domain. The initial positions are defined as

$$
x_{i, 0}=x_{\min }+r\left(x_{\max }-x_{\min }\right),
$$

where $r$ is a random number in the interval $[0,1]$. Considering the upper and lower bounds, the initial velocity of each particle is given as

$$
v_{i, 0}=\frac{x_{\min }+r\left(x_{\max }-x_{\min }\right)}{\delta t},
$$

where $\delta t$ is the time discretization. 
The velocity of each particle is updated according to

$$
v_{i, k+1}=c_{1} v_{i, k}+c_{2} r_{1}\left(p_{i, k}-x_{i, k}\right)+c_{3} r_{2}\left(g_{k}-x_{i, k}\right),
$$

where $k$ stands for the discretized time.

In Eq. (21), the term $c_{1}$ is called the inertia weight and controls the impact of the previous velocity on the current one. The parameters $c_{2}$ and $c_{3}$ are positive constants, called cognitive and social parameters, respectively. The fine tuning of these parameters will help to achieve faster convergence of the PSO algorithm. The parameters $r_{1}$ and $r_{2}$ are two random numbers having values in the interval $[0,1]$. Their role is to keep the population diversity. The parameter $p_{i, k}$ is defined as the best location found by particle $i$ up to time $k$. Finally, the parameter $g_{k}$ is defined as the best global position found among all particles in the swarm up to time $k$.

Finally, the position is updated as

$$
x_{i, k+1}=x_{i, k}+v_{i, k+1} \delta t .
$$

Here, $\delta t=1$ and a new time instant corresponds simply to a new iteration of the algorithm.

The fitness for the locations $p_{i, k}$ and $g_{k}$ is defined as $f_{i \text {,best }}$ and $f_{g \text {,best }}$, respectively. The objective is then to minimize the difference between $f_{i \text {, best }}$ and $f_{g \text {,best }}$ such that no further improvement is introduced. It normally takes from few hundred to few thousand iterations until convergence is achieved. In the present problem, the values for the positions of the particles $\left(x_{i, k}\right)$ are the cross-section areas $\left(A_{i}\right)$ at the iteration $k$.

\subsection{The hybrid approach}

Trying to keep the best features of the Levenberg-Marquardt and the Particle Swarm Optimization methods, a hybrid approach combining both methods is considered in the present work. Recently, hybrid approaches, coupling stochastic methods and the LM one have been successfully used for the solution of inverse problems of parameter estimation [36] as SA-LM (Simulated Annealing and Levenberg-Marquardt) and GA-LM (Genetic Algorithms and Levenberg-Marquardt). Other hybrid strategies combining stochastic and deterministic methods are reported in the literature [42].

Essentially, the adopted procedure is to generate an initial guess for the Levenberg-Marquardt method using the output of the Particle Swarm Optimization one. A well known handicap of the LM method, specially when dealing with noisy data, is the possibility of converging to local minima, stopping before arriving at the desired global minimum. Besides, the convergence of the LM method is greatly dependent on the initial guess for the parameters to be identified. With the PSO method used to provide the initial guess, a better accuracy may be obtained, the actual global minimum is likely to be achieved, and the number of iterations necessary to obtain the minimum is reduced, as it will be seen next.

\section{Identification errors with noiseless data}

\subsection{Identification with the Levenberg-Marquardt method}

The Levenberg-Marquardt method (LM) was used for the identification of the damage scenarios shown in Fig. 1. In all cases, it was firstly considered noiseless data. The LM method did not converge for some of the test cases, even in the absence of noise, and for those that a reasonable solution was achieved, the parameter $\Gamma$ in Eq. (17) had to be adjusted. This fine tuning is very time consuming and problem dependent.

In order to illustrate the influence of the relaxation factor $\Gamma$ in the results provided by the LM method, Fig. 3 depicts the damage estimates obtained for Case 1 (see Fig. 1), with $a=25 \mathrm{~mm}$ and $d=7.5 \mathrm{~mm}$. For convenience, the cross-section area is normalized with respect to the nominal cross-section area of the bar. A total of 31 unknown parameters were estimated, in the present case, with the values $A_{i}=1.0$ as initial guess. It can be observed that for $\Gamma=10000$ a physical meaningless solution is obtained. For $\Gamma=1$ the solution is very close to the initial guess, even after 100 iterations of the algorithm. When $\Gamma=1000$ is used, the solution is improved, but a deviation from the exact solution is still observed even after 100 iterations. When $\Gamma=5000$ the exact solution is recovered with 50 iterations. 


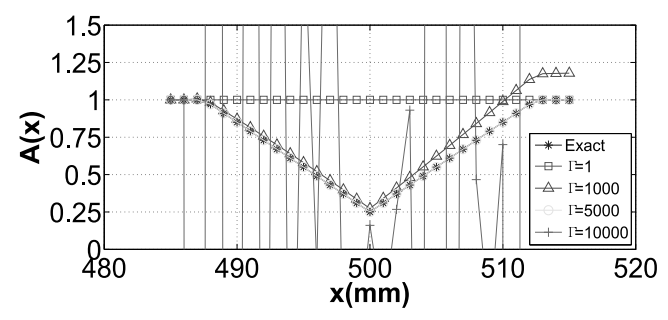

Fig. 3. Effect of the relaxation factor $\Gamma$ on the solution with the LM method.

In order to avoid the difficulties observed with the LM method, it was implemented a hybrid approach in which the PSO method is used to generate an initial guess for LM. In such approach a fixed value of $\Gamma=10000$ was used for all test cases.

All results shown from now on were obtained using the stochastic method PSO and the hybrid approach PSO-LM. The adopted value for the constants $\left(c_{1}, c_{2}, c_{3}\right)$, in all cases, is equal to $0.2, \epsilon=10^{-10}$, and the number of particles is $10^{3}$, as suggested in [3].

\subsection{Identification using the impulse response}

The general procedure for obtaining the inverse problem solution is briefly described as follows. An impulsive wave, $f(t)=\delta(t)$, is given as the input for the direct wave propagation problem, i.e., as a progressive plane wave propagating along the bar, for each one of the studied damages, as described in [40]. Then, the corresponding synthesized echoes are assumed as the experimental impulse responses. In the sequel, the optimization methods (PSO and PSO-LM) are applied, with the aim at updating the cross section area profile of the model in order to fit its predicted echo to the experimental one, for each damage scenario.

Firstly, the PSO method was applied to the problem. This method showed a good convergence but the optimal minimum was not reached in all situations. Then, the hybrid PSO-LM method was considered, improving the final solution.

The relative errors obtained in the identification of the cross section area profile for Cases 1, 3 and 4 (see Fig. 1), considering both optimization methods, are plotted in Fig. 4. It is worth noting that, actually, only the PSO method presented some identification errors, no more than $0.6 \%$, in the absence of noise.

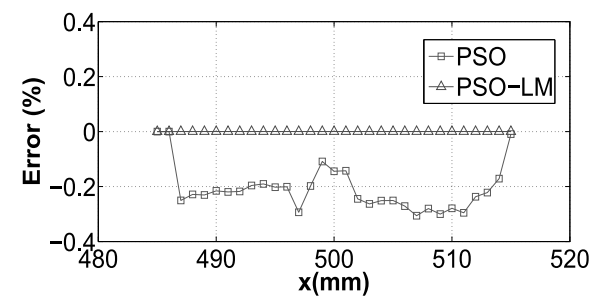

(a) Case 1

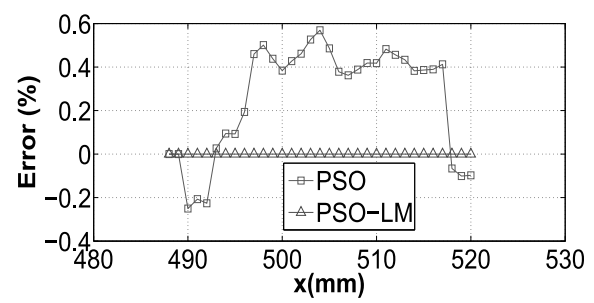

(b) Case 3

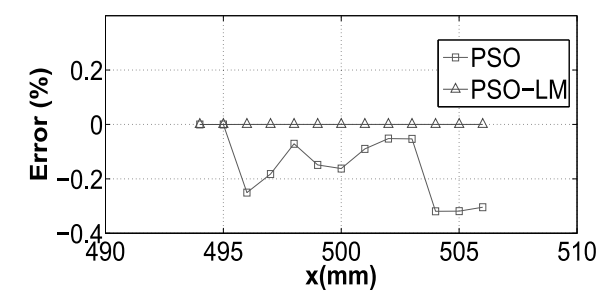

(c) Case 4

Fig. 4. Relative errors in the cross section area identification for Cases 1, 3 and 4. 


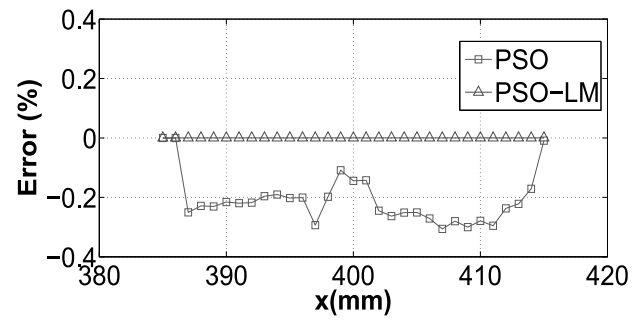

(a) Case 2, first part

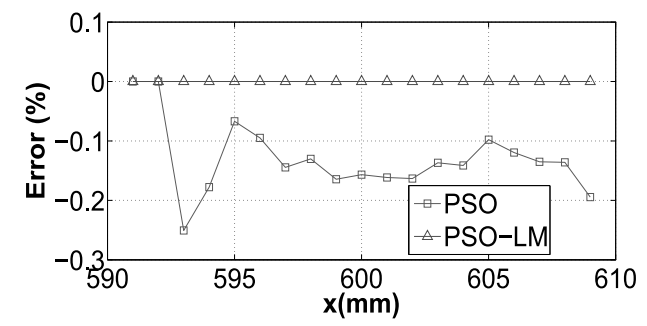

(b) Case 2, second part

Fig. 5. Relative errors in the cross section area identification for Case 2, first and second parts.

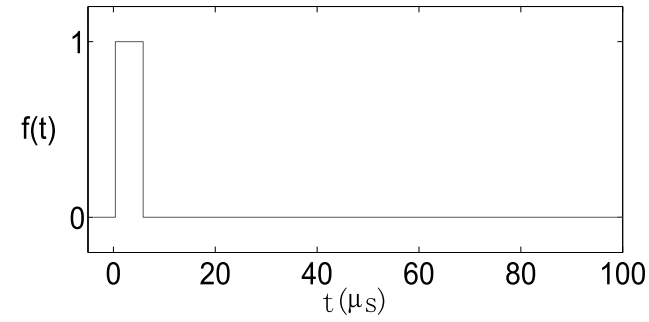

(a) Short rectangular pulse

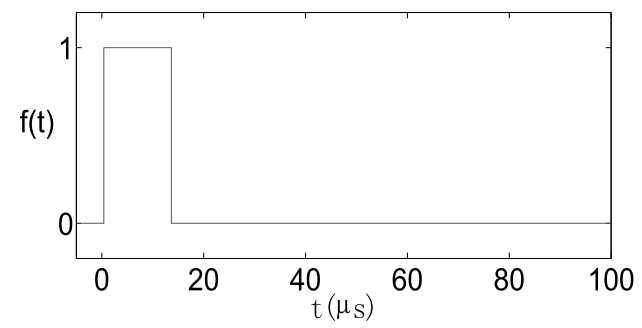

(b) Long rectangular pulse

Fig. 6. Rectangular pulse excitations.

The damage scenario of Case 2, Fig. 1b, is comprised of two damaged regions apart from each other. In this case the first part of the echo is due, only, to the first damage, with no influence of the second one, since the incident wave reaches the first damage without passing by any other inhomogeneity within the bar. On the other hand, the second part of Fig. $2 \mathrm{~b}$ is due to the second damage with the influence of the first one. Therefore, the following damage identification procedure was adopted: The cross section area of the first damaged region was identified from its corresponding part of the echo; then, the second damaged region was estimated using the previously identified first part.

For Case 2, see Fig. 5, the error is presented in two parts, corresponding to the two separated damages in the bar. With the PSO method alone, the results were also quite satisfactory, showing an error lower than $0.3 \%$ in the worst case.

Considering Figs 4 and 5, one may clearly see that the PSO-LM method yielded the more accurate inverse problem solution, in the absence of noise.

\subsection{Identification using rectangular pulse excitation}

The test cases were also performed considering rectangular pulses. Due to its inherent complexity, the damage scenario depicted in Fig. 1c (Case 3) was chosen to evaluate the performance of the identification methods. Two types of rectangular pulses were used. The one shown in Fig. 6a corresponds to an excitation whose support is of the order of the damage length, while the second one, shown in Fig. 6b, has a support that is larger than the damage length. Indeed, the short rectangular pulse is $5 \mu \mathrm{s}$ long, while the long rectangular pulse is $15 \mu \mathrm{s}$ long. Since the wave speed is around $5 \times 10^{3} \mathrm{~m} / \mathrm{s}$, the short pulse has a length of $25 \mathrm{~mm}$, around the same length of the damage in Case 3, see Fig. 1c, while the long rectangular pulse has a length of $75 \mathrm{~mm}$, around three times the damage length.

Figure 7 a shows the damage identification results for Case 3 with the short rectangular pulse and noiseless data. The corresponding relative errors in the identification are presented in Fig. 7b. As one can see from Fig. 7, both methods succeeded in the identification; however the PSO-LM method yielded the exact damage profile, while the PSO method alone, being a stochastic technique, showed a slight random dispersion around the exact profile.

In Fig. 8 the identification results considering the long rectangular pulse excitation are shown. From the results shown in Figs 7 and 8, it is not possible to observe any significant influence of the pulse excitation support on the quality of the identification results, when noiseless data are used. 


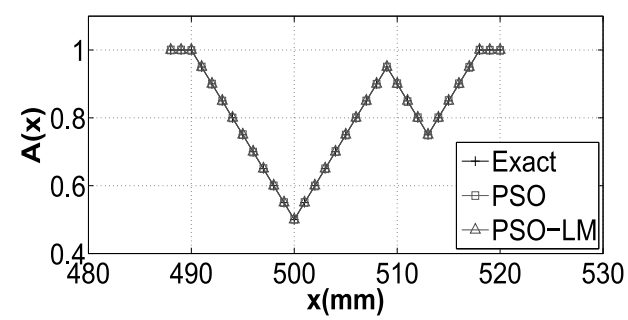

(a) Identification results

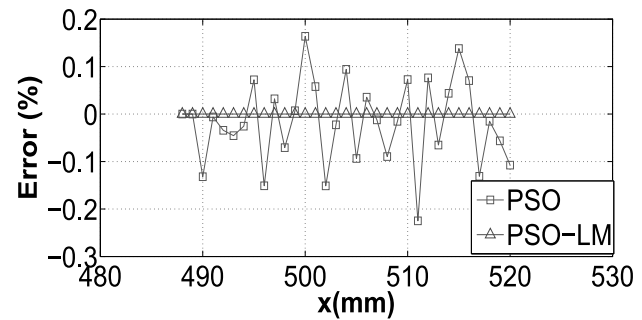

(b) Relative identification errors

Fig. 7. Identification with noiseless data for Case 3 and the short rectangular pulse excitation.

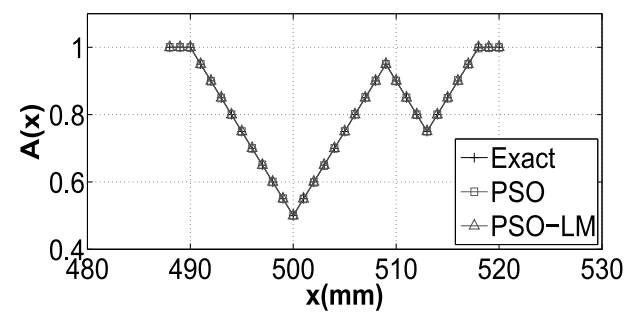

(a) Identification results

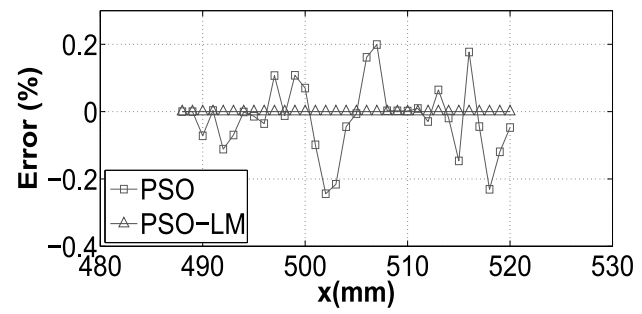

(b) Relative identification errors

Fig. 8. Identification with noiseless data for Case 3 and the long rectangular pulse excitation.

\section{Identification with noisy data}

We will now discuss the identification results with the disturbance of additive noise in both pulse and echo signals.

\subsection{Identification using the impulse response}

In the identification of actual damages with pulse-echo tests, there is always some level of additive noise in the experimental data. In order to verify the influence of distinct levels of signal to noise ratio, SNR, four different levels of zero mean random noise were added to the output signal, corresponding to $\mathrm{SNR}=30,20,10$ and $0 \mathrm{~dB}$. Note that, in this last and worst case, the noise average power is equivalent to the signal average power, a condition which is seldom found in a well controlled experimental setup.

Figure 9 shows the impulse responses for the damaged structure for Case 1, see Fig. 1a, with $a=25 \mathrm{~mm}$ and $d=5 \mathrm{~mm}$, corrupted with random noise, corresponding to SNR's of 30,20, 10, and $0 \mathrm{~dB}$.

Figure 10 presents the identification results provided by the PSO and PSO-LM methods, along with the actual damage profile. As can be observed, there is no noticeable difference among the three curves - exact profile and identified ones - for SNR of 30 and $20 \mathrm{~dB}$. However, for greater noise levels (SNR of 10 and $0 \mathrm{~dB}$ ), slight errors are observed in the results provided by the two identification methods. Since this behavior remains the same for all other tested damage scenarios, in the figures that follow, only the greater noise levels (SNR of 10 and $0 \mathrm{~dB}$ ) will be depicted.

Figure 11 shows the effect of the damage parameters $a$ and $d$ on the identification of the triangular profile of Case 1, in the presence of the higher noise level in the synthetic data, $\mathrm{SNR}=0 \mathrm{~dB}$. Four different damage intensities were obtained through the variation of these parameters. In Figs 11a and 11b, the damage depth $d$, which represents the damage severity, is changed. On the other hand, in Figs 11c and 11d, a modification in the damage length $a$ is analysed. Comparing Figs $10 \mathrm{~d}$ and Fig. 11, both with SNR $=0 \mathrm{~dB}$, it is worth noting that changes in the damage parameters $a$ and $d$ do not alter the capability of the method in identifying the damage profile. However, in the least severe case, shown in Fig. 11a, the relative identification error is slightly greater, as expected.

Figure 12 depicts the identification of the cross section area profile of the first damaged region (Part 1) of Case 2. The identification result for the second damaged region (Part 2) is depicted in Fig. 13. 


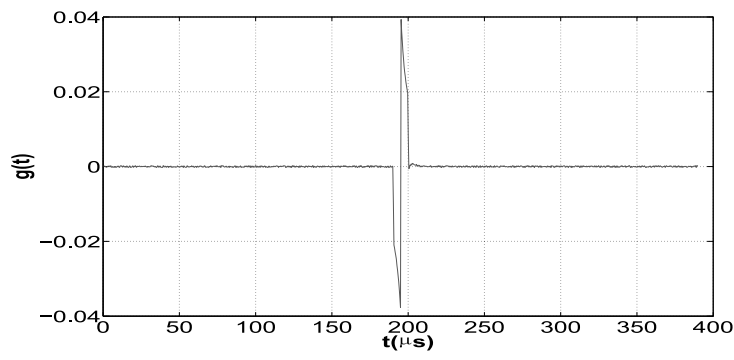

(a) $\mathrm{SNR}=30 \mathrm{~dB}$

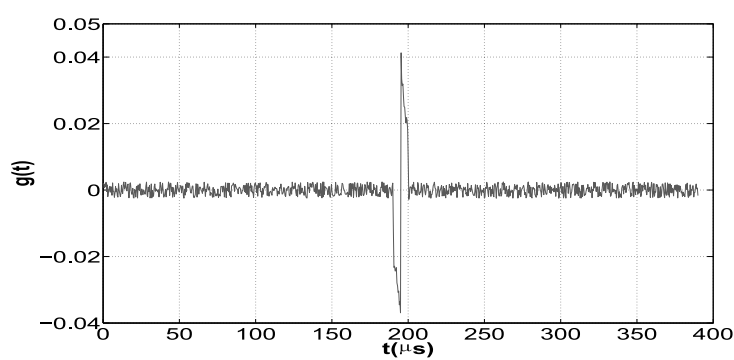

(c) $\mathrm{SNR}=10 \mathrm{~dB}$

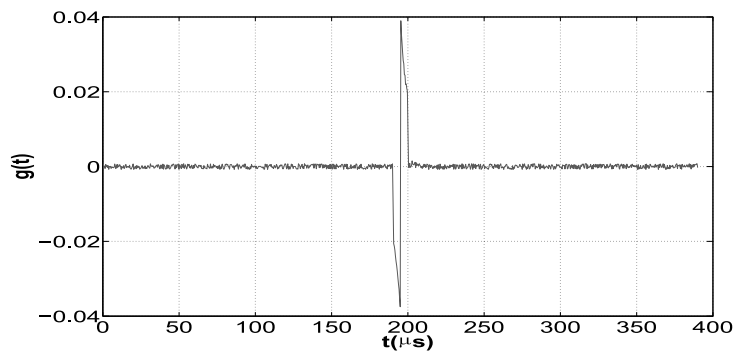

(b) $\mathrm{SNR}=20 \mathrm{~dB}$

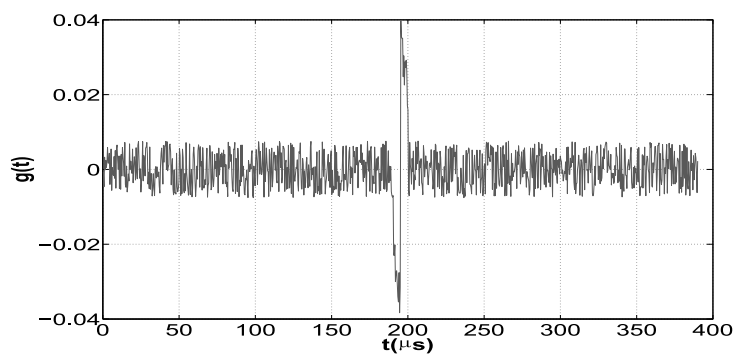

(d) $\mathrm{SNR}=0 \mathrm{~dB}$

Fig. 9. Impulse responses for Case 1, with $a=25 \mathrm{~mm}$ and $d=5 \mathrm{~mm}$, with different levels of SNR.

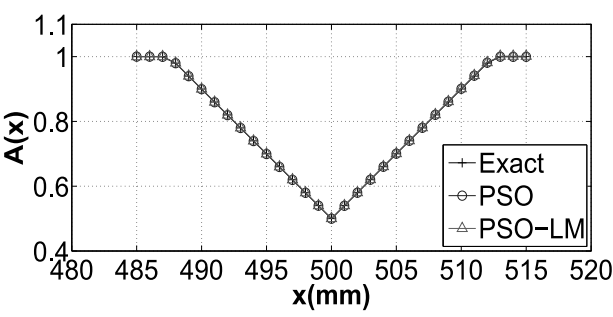

(a) $\mathrm{SNR}=30 \mathrm{~dB}$

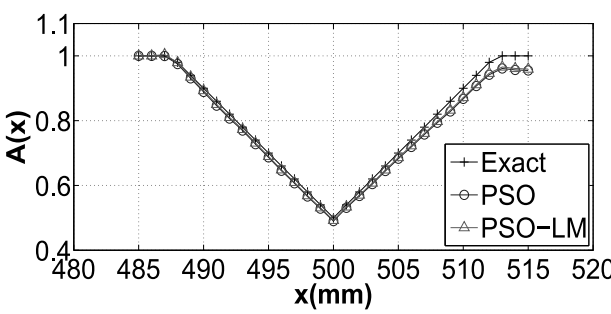

(c) $\mathrm{SNR}=10 \mathrm{~dB}$

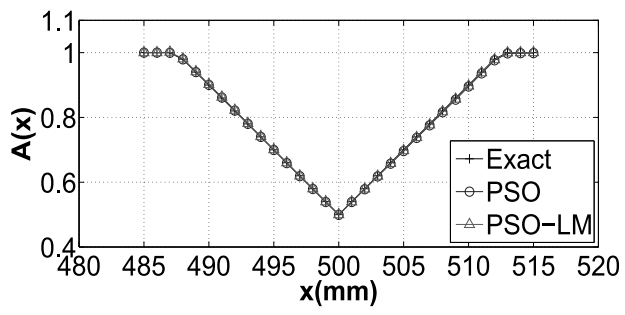

(b) $\mathrm{SNR}=20 \mathrm{~dB}$

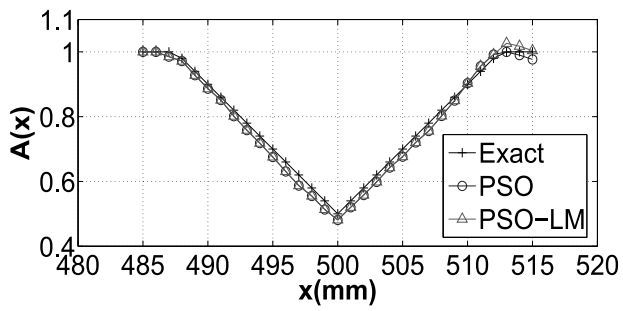

(d) SNR $=0 \mathrm{~dB}$

Fig. 10. Damage identification with four levels of SNR, Case 1, $a=25 \mathrm{~mm}$ and $d=5 \mathrm{~mm}$.

The profile identification for Case 3, with two levels of SNR (10 and $0 \mathrm{~dB})$ is depicted in Fig. 14. From the results provided by the two methods, it is worth noting that the damage profile is clearly identified.

Finally, Fig. 15 presents the identification results for Case 4. Note that, since plane wave propagation is considered, the actual circular shape of the damage (see Fig. 1d) and its position in the vertical axis are not recognizable, but only the corresponding cross section area variation of the bar can be assessed.

Table 1 illustrates the main figures related to the damage identification for Cases 1 to 4 with the hybrid PSO-LM method: Number of synthetic experimental data used in the identification; number of estimated parameters; number of iteractions for four SNR levels; and the residue of the estimation process, Eq. (15). It shows that very good results 
Table 1

Main figures for the hybrid PSO-LM method

\begin{tabular}{lccccc}
\hline & Case 1 & Case 2 (Part 1) & Case 2 (Part 2) & Case 3 & Case 4 \\
\hline Synthetic data & 31 & 31 & 19 & 33 & 13 \\
Estimated parameters & 29 & 29 & 17 & 31 & 11 \\
Iterations $(0 \mathrm{~dB})$ & 34 & 32 & 21 & 39 & 14 \\
Iterations $(10 \mathrm{~dB})$ & 33 & 31 & 21 & 38 & 14 \\
Iterations $(20 \mathrm{~dB})$ & 33 & 34 & 22 & 39 & 13 \\
Iterations $(30 \mathrm{~dB})$ & 31 & 34 & 21 & 35 & 14 \\
Error $(0 \mathrm{~dB})$ & $1.669 \mathrm{E}^{-14}$ & $1.805 \mathrm{E}^{-14}$ & $3.703 \mathrm{E}^{-15}$ & $2.088 \mathrm{E}^{-14}$ & $8.833 \mathrm{E}^{-16}$ \\
Error $(10 \mathrm{~dB})$ & $1.833 \mathrm{E}^{-14}$ & $1.671 \mathrm{E}^{-14}$ & $3.370 \mathrm{E}^{-15}$ & $1.957 \mathrm{E}^{-14}$ & $8.570 \mathrm{E}^{-16}$ \\
Error $(20 \mathrm{~dB})$ & $1.710 \mathrm{E}^{-14}$ & $1.706 \mathrm{E}^{-14}$ & $3.400 \mathrm{E}^{-15}$ & $2.043 \mathrm{E}^{-14}$ & $8.383 \mathrm{E}^{-16}$ \\
Error $(30 \mathrm{~dB})$ & $1.711 \mathrm{E}^{-14}$ & $1.717 \mathrm{E}^{-14}$ & $3.486 \mathrm{E}^{-15}$ & $2.019 \mathrm{E}^{-14}$ & $8.187 \mathrm{E}^{-16}$ \\
\hline
\end{tabular}

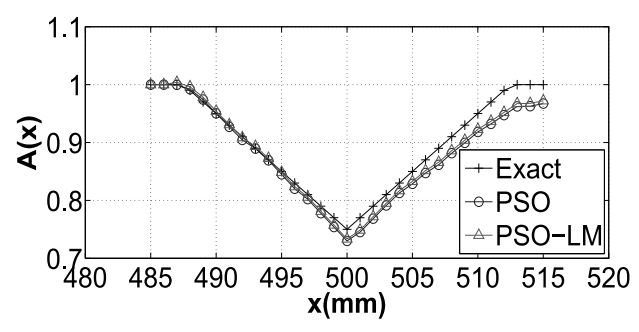

(a) $\mathrm{a}=25 \mathrm{~mm}, \mathrm{~d}=2.5 \mathrm{~mm}$

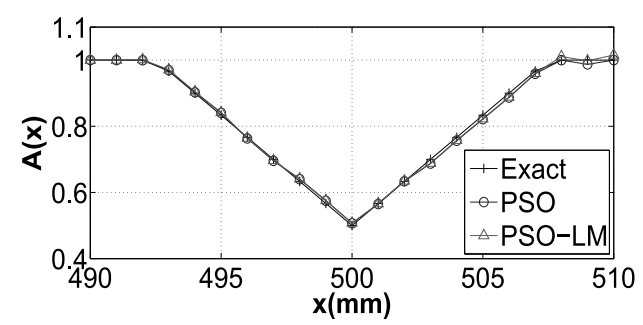

(c) $\mathrm{a}=15 \mathrm{~mm}, \mathrm{~d}=5 \mathrm{~mm}$

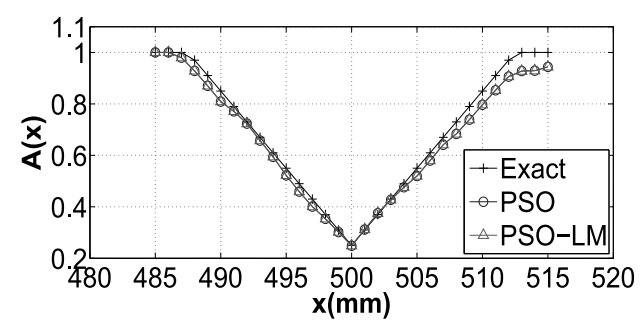

(b) $\mathrm{a}=25 \mathrm{~mm}, \mathrm{~d}=7.5 \mathrm{~mm}$

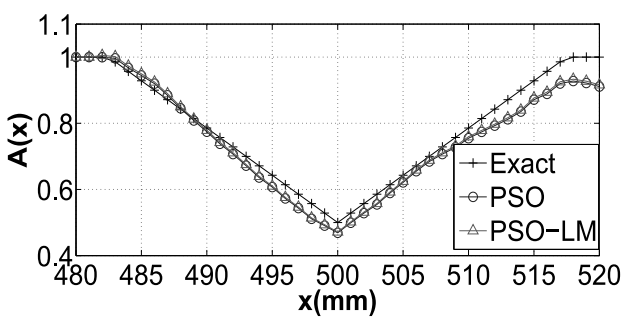

(d) $\mathrm{a}=35 \mathrm{~mm}, \mathrm{~d}=5 \mathrm{~mm}$

Fig. 11. Damage identification for different values of $a$ and $d$, Case $1, \mathrm{SNR}=0 \mathrm{~dB}$.

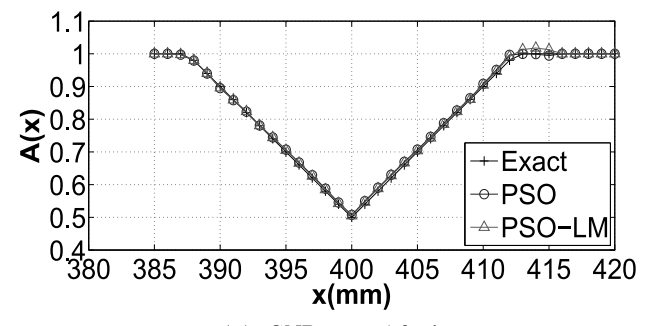

(a) $\mathrm{SNR}=10 \mathrm{~dB}$

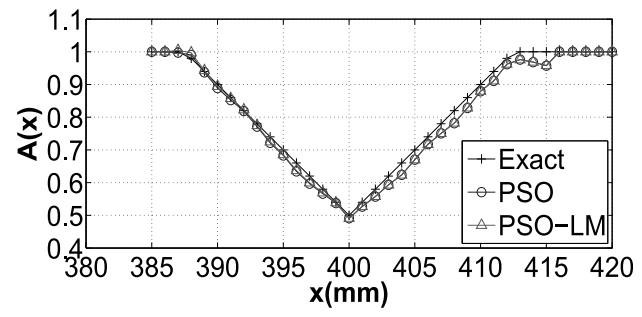

(b) $\mathrm{SNR}=0 \mathrm{~dB}$

Fig. 12. Damage identification with two levels of SNR, Case 2 (Part 1).

were obtained with the hybrid PSO-LM method with regard to the number of iterations and the achieved minimum value of the cost function.

Tables 2 and 3 presents a comparison of the computational burden of the PSO and PSO-LM methods for the four test cases and different signal to noise ratios. As can be seen, the computational effort of the PSO method alone is much greater than that of the PSO-LM method. For instance, in Case 4 with SNR of $20 \mathrm{~dB}$, computational time with the PSO method was about 1 hour and with the PSO-LM method it was around 10 seconds. The reason for 
Table 2

Computational effort for the PSO method

\begin{tabular}{lccccc}
\hline & Case 1 & $\begin{array}{c}\text { Case 2 } \\
\text { (Part 1) }\end{array}$ & $\begin{array}{c}\text { Case 2 } \\
\text { (Part 2) }\end{array}$ & Case 3 & Case 4 \\
\hline Computational time (0 dB) & $3 \mathrm{~h} 47 \mathrm{~m} 24 \mathrm{~s}$ & $3 \mathrm{~h} 45 \mathrm{~m} 00 \mathrm{~s}$ & $3 \mathrm{~h} 52 \mathrm{~m} 42 \mathrm{~s}$ & $8 \mathrm{~h} 20 \mathrm{~m} 24 \mathrm{~s}$ & $3 \mathrm{~m} 13 \mathrm{~s}$ \\
Executions - SAA $(0 \mathrm{~dB})$ & $6,824,000$ & $3,541,000$ & 128,000 & $5,000,000$ & 66,000 \\
Computational time $(10 \mathrm{~dB})$ & $3 \mathrm{~h} 23 \mathrm{~m} 24 \mathrm{~s}$ & $3 \mathrm{~h} 46 \mathrm{~m} 48 \mathrm{~s}$ & $9 \mathrm{~h} 34 \mathrm{~m} 12 \mathrm{~s}$ & $1 \mathrm{~h} 53 \mathrm{~m} 24 \mathrm{~s}$ & $58 \mathrm{~m} 23 \mathrm{~s}$ \\
Executions - SAA $(10 \mathrm{~dB})$ & $5,278,000$ & $3,516,000$ & 483,000 & $1,049,000$ & 901,000 \\
Computational time $(20 \mathrm{~dB})$ & $2 \mathrm{~h} 11 \mathrm{~m} 24 \mathrm{~s}$ & $3 \mathrm{~h} 28 \mathrm{~m} 48 \mathrm{~s}$ & $4 \mathrm{~h} 48 \mathrm{~m} 00 \mathrm{~s}$ & $6 \mathrm{~h} 20 \mathrm{~m} 22 \mathrm{~s}$ & $1 \mathrm{~h} 0036 \mathrm{~s}$ \\
Executions - SAA $(20 \mathrm{~dB})$ & $2,702,000$ & $1,796,000$ & 191,000 & $4,491,000$ & 901,000 \\
Computational time $(30 \mathrm{~dB})$ & $2 \mathrm{~h} 09 \mathrm{~m} 36 \mathrm{~s}$ & $4 \mathrm{~h} 10 \mathrm{~m} 45 \mathrm{~s}$ & $4 \mathrm{~h} 03 \mathrm{~m} 00 \mathrm{~s}$ & $3 \mathrm{~h} 43 \mathrm{~m} 48 \mathrm{~s}$ & $56 \mathrm{~m} 56 \mathrm{~s}$ \\
Executions - SAA $(30 \mathrm{~dB})$ & $2,689,000$ & $3,565,000$ & 183,000 & $3,495,000$ & 897,000 \\
\hline
\end{tabular}

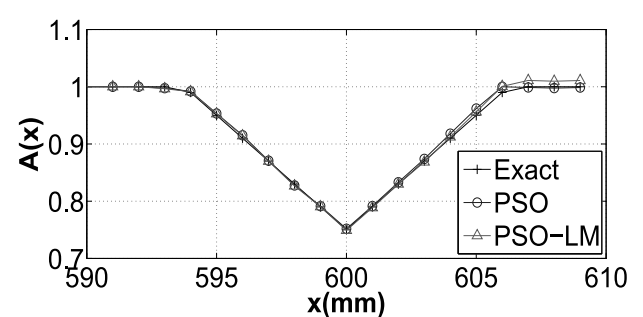

(a) $\mathrm{SNR}=10 \mathrm{~dB}$

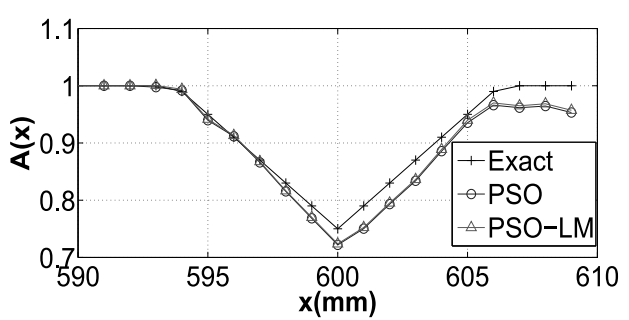

(b) $\mathrm{SNR}=0 \mathrm{~dB}$

Fig. 13. Damage identification with two levels of SNR, Case 2 (Part 2).

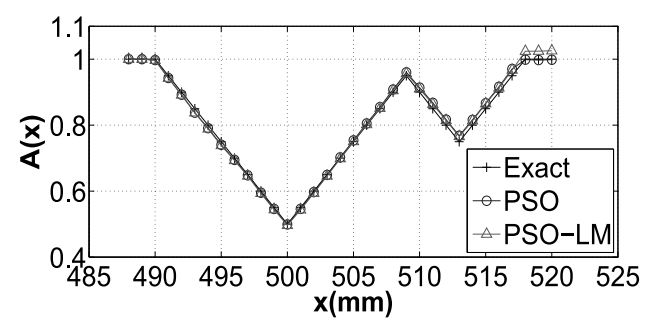

(a) $\mathrm{SNR}=10 \mathrm{~dB}$

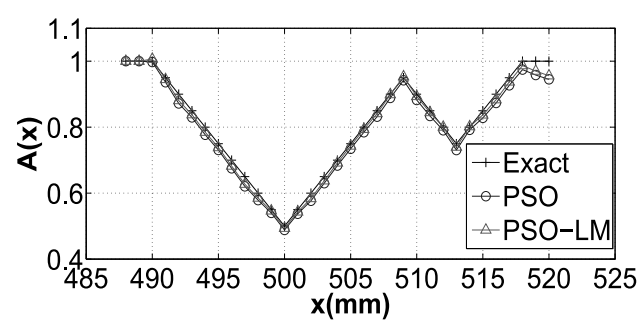

(b) $\mathrm{SNR}=0 \mathrm{~dB}$

Fig. 14. Damage identification with two levels of SNR, Case 3.

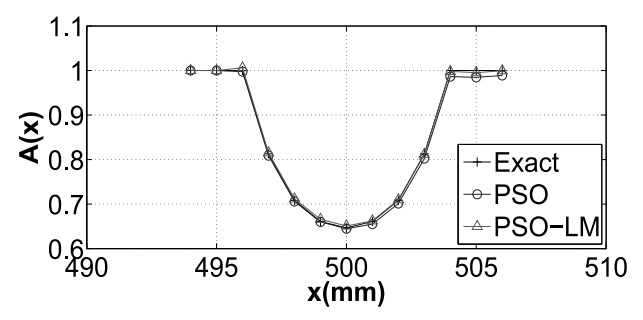

(a) $\mathrm{SNR}=10 \mathrm{~dB}$

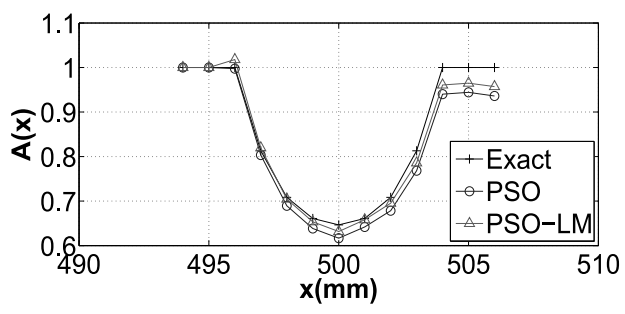

(b) $\mathrm{SNR}=0 \mathrm{~dB}$

Fig. 15. Damage identification with two levels of SNR, Case 4.

this strong discrepancy comes from the stochastic nature of the PSO, which demands a relatively great number of iterations to reach a small residue value. On the other hand, in the hybrid PSO-LM approach, the PSO algorithm runs only up to a specified number of iterations in order to provide the initial guess for the deterministic LM algorithm, which runs relatively fast. 
Table 3

Computational effort for the PSO-LM method

\begin{tabular}{lccccc}
\hline & Case 1 & $\begin{array}{c}\text { Case 2 } \\
(\text { Part 1) }\end{array}$ & $\begin{array}{c}\text { Case 2 } \\
\text { (Part 2) }\end{array}$ & Case 3 & Case 4 \\
\hline Computational time $(0 \mathrm{~dB})$ & $3 \mathrm{~m} 46 \mathrm{~s}$ & $2 \mathrm{~m} 48 \mathrm{~s}$ & $15 \mathrm{~m} 15 \mathrm{~s}$ & $5 \mathrm{~m} 4 \mathrm{~s}$ & $9.72 \mathrm{~s}$ \\
Executions - SAA $(0 \mathrm{~dB})$ & 57,223 & 53,857 & 12,160 & 74,998 & 3,403 \\
Computational time $(10 \mathrm{~dB})$ & $3 \mathrm{~m} 10 \mathrm{~s}$ & $2 \mathrm{~m} 40 \mathrm{~s}$ & $15 \mathrm{~m} 01 \mathrm{~s}$ & $3 \mathrm{~m} 53 \mathrm{~s}$ & $10.24 \mathrm{~s}$ \\
Executions - SAA $(10 \mathrm{~dB})$ & 55,540 & 52,174 & 12,160 & 73,075 & 3,403 \\
Computational time $(20 \mathrm{~dB})$ & $2 \mathrm{~m} 55 \mathrm{~s}$ & $2 \mathrm{~m} 57 \mathrm{~s}$ & $15 \mathrm{~m} 50 \mathrm{~s}$ & $4 \mathrm{~m} 7 \mathrm{~s}$ & $9.94 \mathrm{~s}$ \\
Executions - SAA $(20 \mathrm{~dB})$ & 55,540 & 57,223 & 12,739 & 74,998 & 3,160 \\
Computational time $(30 \mathrm{~dB})$ & $2 \mathrm{~m} 39 \mathrm{~s}$ & $3 \mathrm{~m} 11 \mathrm{~s}$ & $16 \mathrm{~m} 15 \mathrm{~s}$ & $3 \mathrm{~m} 43 \mathrm{~s}$ & $10.58 \mathrm{~s}$ \\
Executions - SAA $(30 \mathrm{~dB})$ & 52,174 & 57,223 & 12,160 & 67,306 & 3,403 \\
\hline
\end{tabular}

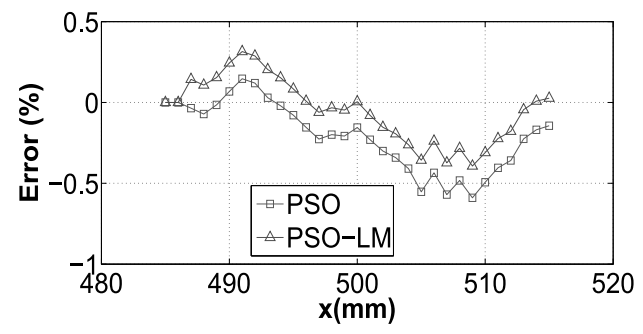

(a) $\mathrm{SNR}=20 \mathrm{~dB}$

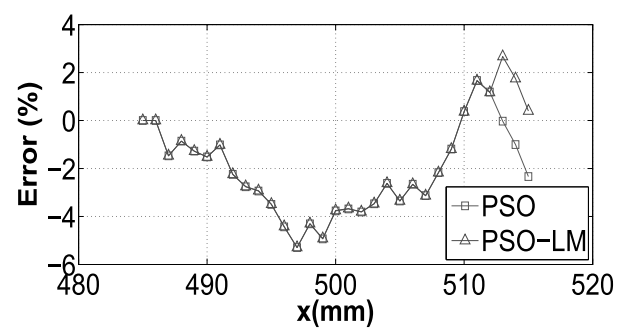

(b) $\mathrm{SNR}=0 \mathrm{~dB}$

Fig. 16. Relative error in the identification for a low $(0 \mathrm{~dB})$ and moderate $(20 \mathrm{~dB})$ levels of SNR, Case $1, a=25 \mathrm{~mm}$ and $d=5.0 \mathrm{~mm}$.

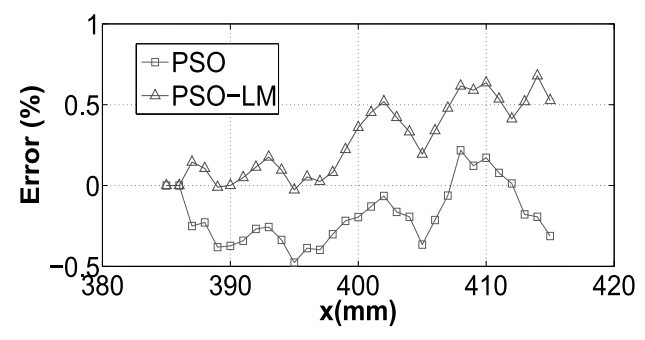

(a) $\mathrm{SNR}=20 \mathrm{~dB}$

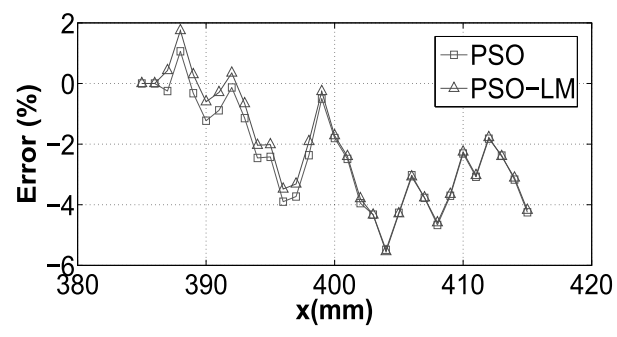

(b) $\mathrm{SNR}=0 \mathrm{~dB}$

Fig. 17. Relative error in the identification for a low $(0 \mathrm{~dB})$ and moderate $(20 \mathrm{~dB})$ levels of SNR, Case 2 (Part 1$)$.

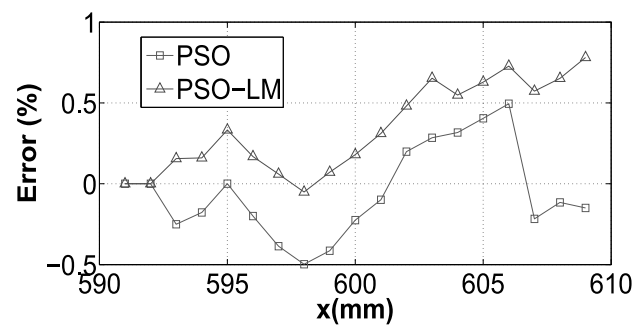

(a) $\mathrm{SNR}=20 \mathrm{~dB}$

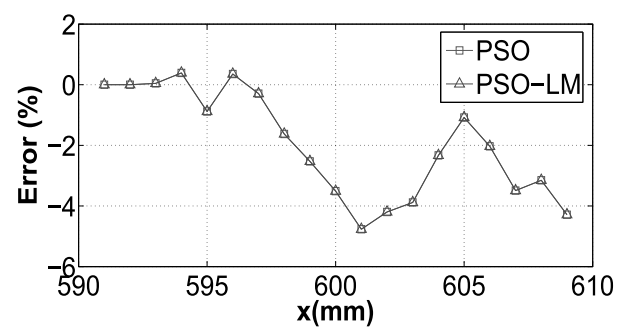

(b) $\mathrm{SNR}=0 \mathrm{~dB}$

Fig. 18. Relative error in the identification for a low $(0 \mathrm{~dB})$ and moderate $(20 \mathrm{~dB})$ levels of SNR, Case 2 (Part 2).

\subsubsection{Relative errors}

In Figs 16 to 20, the relative errors in the damage identification for Cases 1 to 4, using both the PSO and PSO-LM methods are shown. The errors were computed for moderate and high noise levels, SNR of 20 and $0 \mathrm{~dB}$, respectively. 


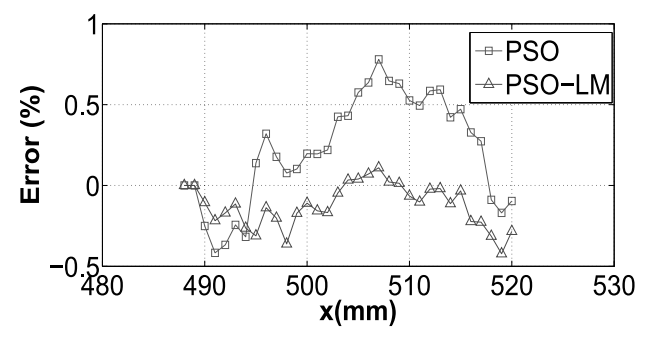

(a) $\mathrm{SNR}=20 \mathrm{~dB}$

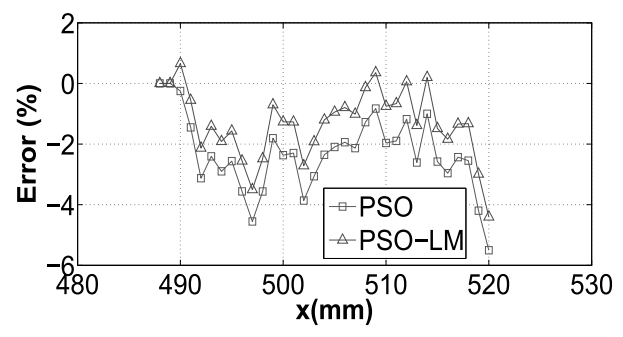

(b) $\mathrm{SNR}=0 \mathrm{~dB}$

Fig. 19. Relative error in the identification for a low $(0 \mathrm{~dB})$ and moderate $(20 \mathrm{~dB})$ levels of SNR, Case 3.

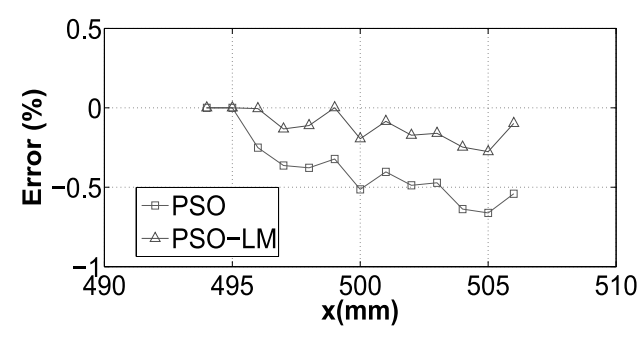

(a) $\mathrm{SNR}=20 \mathrm{~dB}$

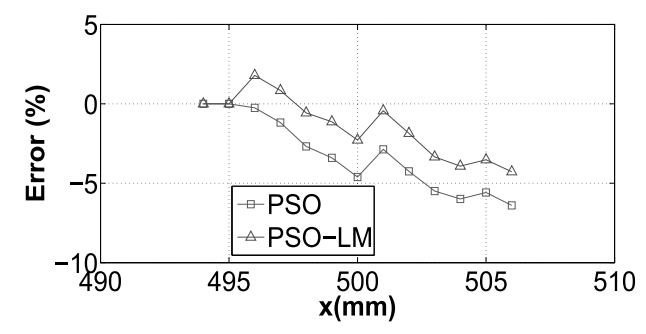

(b) $\mathrm{SNR}=0 \mathrm{~dB}$

Fig. 20. Relative error in the identification for a low $(0 \mathrm{~dB})$ and moderate $(20 \mathrm{~dB})$ levels of SNR, Case 4.

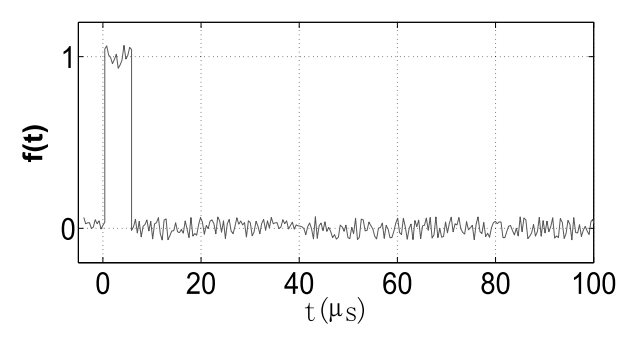

(a) Excitation with SNR $=10 \mathrm{~dB}$

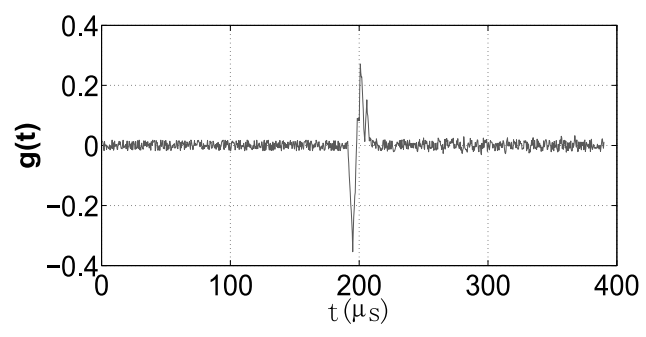

(b) Echo with SNR $=10 \mathrm{~dB}$

Fig. 21. Short rectangular incident pulse and corresponding echo, SNR $=10 \mathrm{~dB}$.

As can be observed from Figs 16 to 20, with SNR of $20 \mathrm{~dB}$ the relative errors, for both methods, are lower than $1 \%$ for all considered cases. As expected, the relative errors increase with SNR of $0 \mathrm{~dB}$, showing to be lower than $5 \%$ for all considered cases. It is worth noting that, in Cases 1 and 2, for the SNR of $0 \mathrm{~dB}$, the relative error presented by both methods are quite the same. However, as it was discussed in Subsection 5.1, the computational effort of the PSO-LM method is considerably lower.

\subsection{Identification with rectangular pulse excitation}

In this section the effect of additive noise in both the rectangular pulse excitation and the echo is considered in the inverse problem procedure. The damage scenario shown in Fig. 1c (Case 3) is taken into account for the test cases. A SNR of $10 \mathrm{~dB}$ - a high level of noise in a measurement setup - is adopted.

In Figs 21 and 22 the rectangular excitations, short and long, respectively, and its respective echoes, both added with random noise with SNR of $10 \mathrm{~dB}$ are shown.

In Figs 23 and 24 the identification results obtained with the PSO and PSO-LM methods, considering the two noisy rectangular excitations are shown. From these figures, it can be observed that, for both rectangular pulses, the damage was satisfactorily identified, with the relative error remaining below $4 \%$. However, a slightly better 


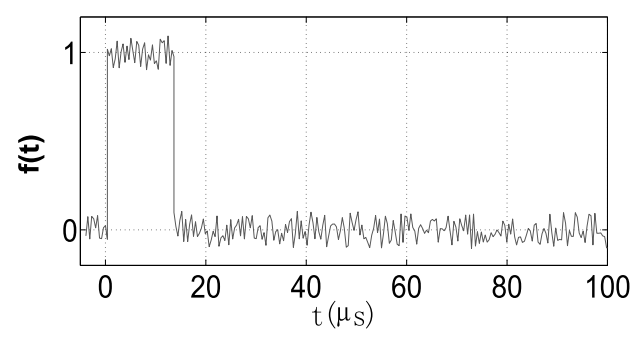

(a) Excitation with SNR $=10 \mathrm{~dB}$

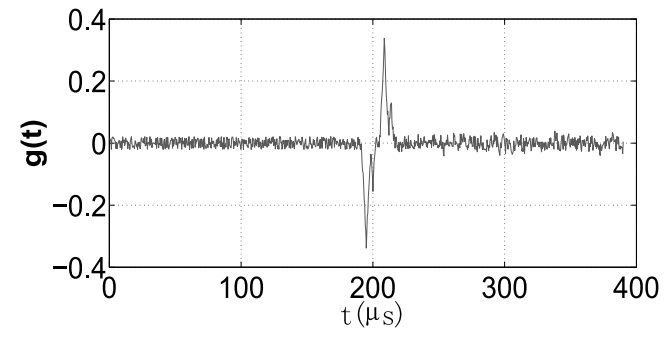

(b) Echo with SNR $=10 \mathrm{~dB}$

Fig. 22. Long rectangular incident pulse and corresponding echo, $\mathrm{SNR}=10 \mathrm{~dB}$.

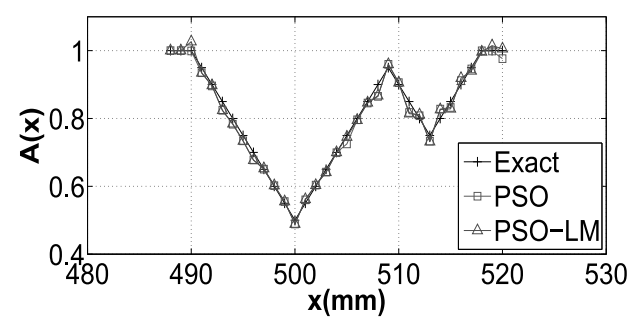

(a) Identification result

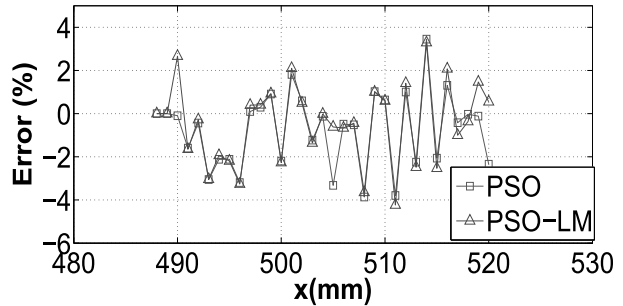

(b) Relative identification error

Fig. 23. Identification with $\mathrm{SNR}=10 \mathrm{~dB}$ for Case 3 and short rectangular pulse.

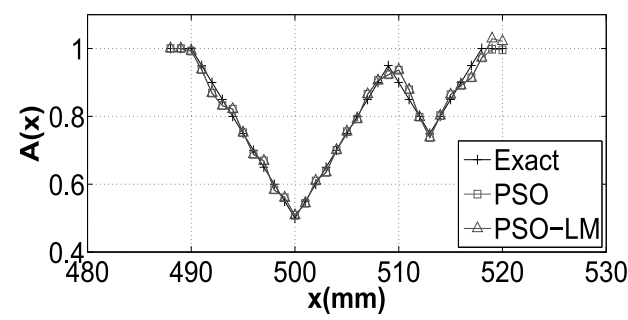

(a) Identification result

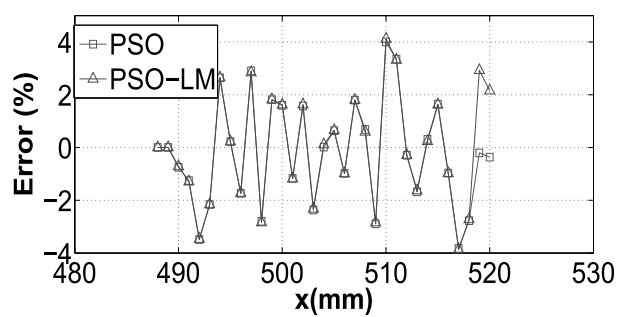

(b) Relative identification error

Fig. 24. Identification with $\mathrm{SNR}=10 \mathrm{~dB}$ for Case 3 and long rectangular pulse.

identification was achieved with the short rectangular pulse. This result is expected since, as it is well known, the Dirac's delta, which provides the impulse response of the damaged bar, extract all required information for the subsequent procedure. On the other hand, short rectangular pulses are closer to the delta, that means, provide higher frequency contents than longer rectangular pulses.

\subsection{Effect of modeling errors}

To verify the influence of an uncertainty in the physical parameters of the structure, an error was inserted in its elastic modulus, which yielded an error in the estimative of the longitudinal wave speed $c$. In the example shown in this section an error of $\pm 10 \%$ in $c$ was considered in the damage identification of Case 1 . The synthetic data were obtained with the SAA method, using the true value of the parameter and considering a SNR of $20 \mathrm{~dB}$, Fig. $9 \mathrm{~b}$. The inverse problem, on the other hand, was solved considering the error in the parameter $c$.

Figure 25 depicts the identification results obtained for $c=0.9 c_{0}, c=c_{0}$ and $c=1.1 c_{0}$, where $c_{0}$ is the actual wave speed. The curve in the center depicts the actual damage and the PSO-LM identification with a SNR of 20 $\mathrm{dB}$, with $c=c_{0}$. The left and right curves represent the identification results obtained with the error of $\pm 10 \%$ in $c$. As expected, an error in the wave speed results in a shift in the identified damage position and also in a change on 


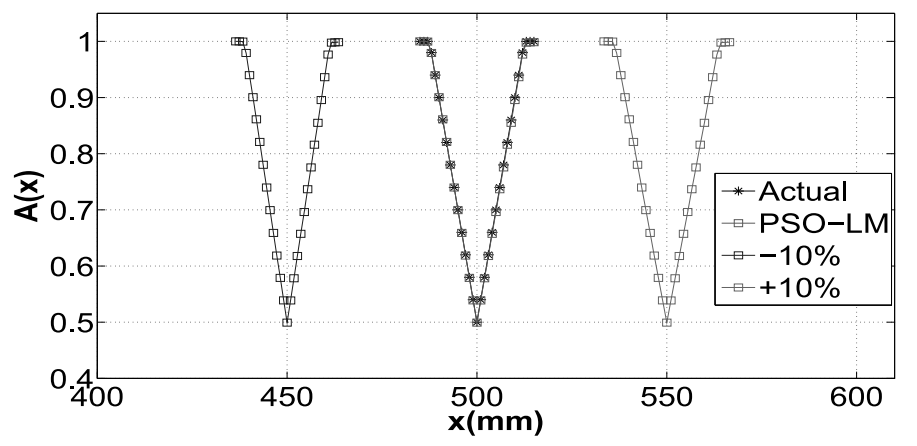

Fig. 25. Effect of modeling errors in the damage identification.

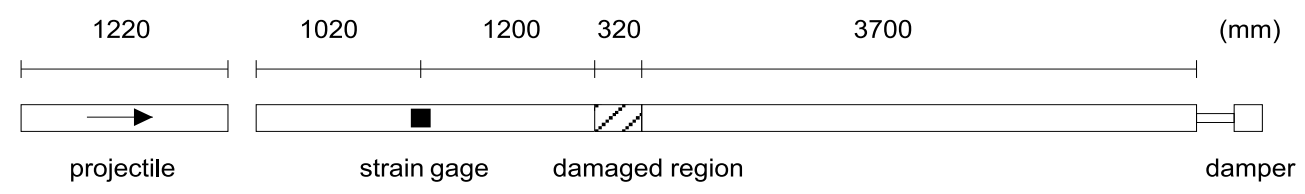

Fig. 26. Experimental pulse-echo setup. Dimensions are in millimeters.

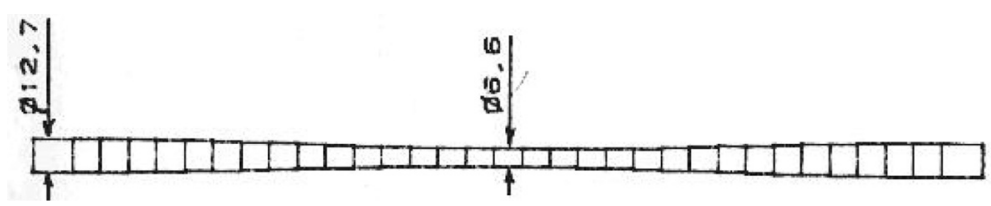

Fig. 27. Machined damage profile. The maximum diameter is the nominal diameter of the bar, $12.7 \mathrm{~mm}$, and the minimum diameter is $6.6 \mathrm{~mm}$.

its length. An error of $+10 \%$ in $c$ shifts the damage center to the left position (the echo was supposed to reach the damage earlier), while an error of $-10 \%$ in $c$ shifts the damage center to the right position (the echo was supposed to reach the damage later), as shown. Furthermore, it is worth noting that the identified damage in the right is $20 \%$ longer than the one in the left. However, as it can be observed, the damage severity (the depth) remains unchanged.

\section{Experimental validation}

In this section, an experimental validation of the sequential algebraic algorithm (SAA), presented in Section 2.1, and, consequently, of the identification procedure is presented. A pulse-echo test in a long circular bar with a limited damaged region is reported. The damage was introduced via the cross-section variation of the bar, as discussed in the numerical examples.

The experimental apparatus consists of an improved Hopkinson bar, depicted in Fig. 26. A 1/2 inch diameter stainless steel continuous bar with $6240 \mathrm{~mm}$ in length is considered for the experimental test. The strain-gage sensor is placed $1020 \mathrm{~mm}$ from the free left end, and a damaged region with $320 \mathrm{~mm}$ in length was machined on it, beginning at $2220 \mathrm{~mm}$ from the free end, as shown in Fig. 26. The machined profile is depicted in Fig. 27. The profile $A(x)$ is a piecewise constant one, each step with $10 \mathrm{~mm}$ in length. At the right end, there is a damper that provides the absorption of the impact energy.

The mechanical impact is produced by the collision of a smaller $1220 \mathrm{~mm}$ bar (the projectile), with the same diameter, at the left end of the bar under test. This impact produces a rectangular-shaped incident progressive wave that travels along the test bar, with a constant speed $c=5104 \mathrm{~m} / \mathrm{s}$, crossing the strain gage (the sensor) station and reaching the damaged region. The acoustic impedance variation due to the cross-section area profile generates a regressive wave that crosses again the strain-gage station. Both the input wave (pulse) and the output wave (echo) signals are measured by the sensor station, as shown in Fig. 28. 


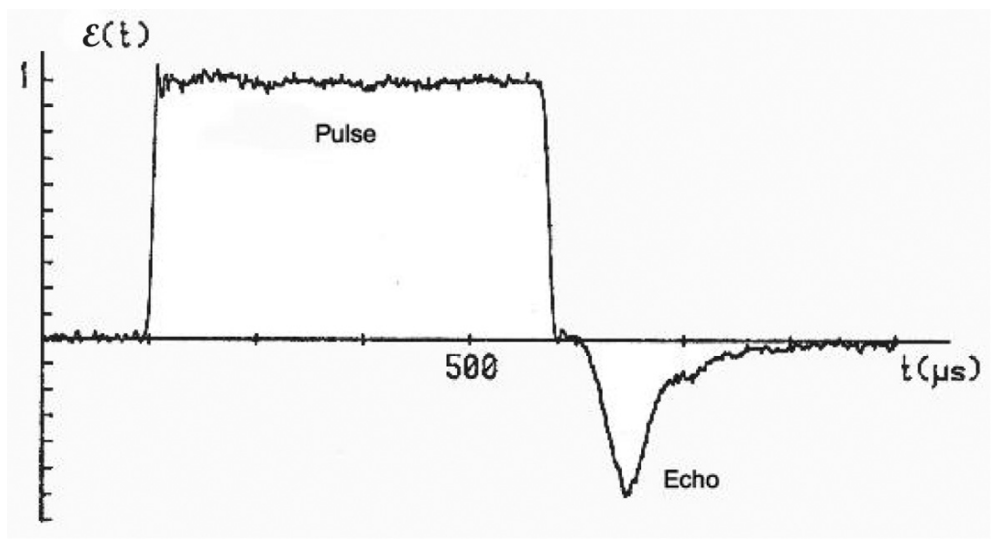

Fig. 28. Signal of pulse and echo measured at the strain gage station.

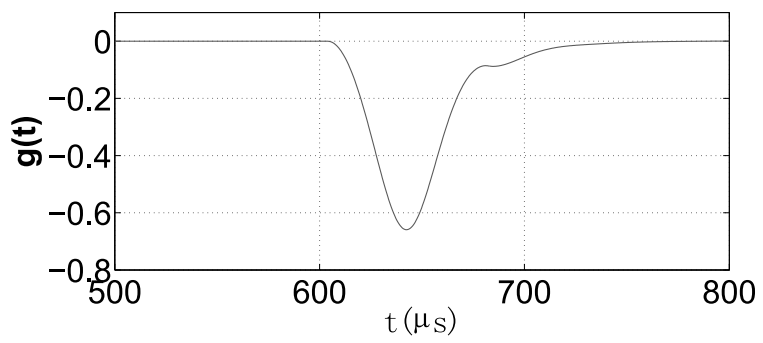

Fig. 29. Echo predicted by the SAA model for the experimental damage.

It is worth noting that the distances shown in Fig. 26 were designed in such a way that the incident pulse completely crosses the pickup station before the arrival of the reflected wavefront and, also, the main part of the echo is acquired before the arrival of the reflected wave due to the boundary condition by the right end of the bar. The damage was machined in the cylindrical bar itself in order to prevent the non-controlled surface contact between, for instance, two bar sections, which provides unknown actual acoustic impedance.

In Fig. 28 it is shown a typical result of the normalized strain signal at the gage station. A compressive (taken as positive) rectangular-shaped incident pulse with a $478 \mu$ s support, corresponding to the collision duration, is followed by a tractive (negative) reflected wave, generated by the damage depicted in Fig. 27. The actual total support of the echo is long, since there is a reverberation phenomenon inside the damaged region, but the portion of it necessary to identify the damage is the one shown in Fig. 28.

The synthetic echo, using the SAA model, for the experimental damage is presented in Fig. 29. The main difference between the synthetic and experimental echoes is that this last one presents some additive noise, characteristic of the experimental setup. But the general shape of the whole echo is the same, as it can be seen by comparing Figs 28 and 29.

In Fig. 30 the identified profile is compared with the actual one, showing not only that the SAA model applies pretty well but also that the hybrid identification technique presented here provides reliable results.

\section{Conclusions}

The inverse problem of damage assessment in a bar using a longitudinal plane wave propagation approach was presented. The damage state of the structure was assessed through the identification of its cross section area profile by using two optimization methods: The stochastic PSO method and a hybrid PSO-LM technique. The LM method alone requires an ad hoc value for the relaxation parameter $\Gamma$, as shown in Fig. 3, therefore it was not considered for identification purposes. 


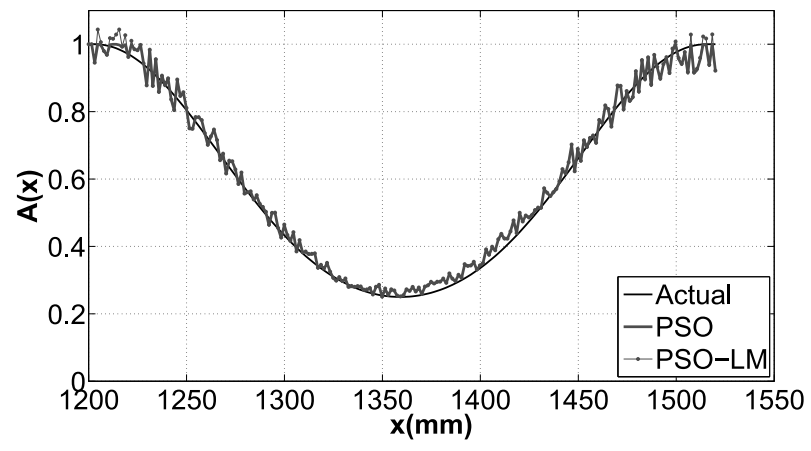

Fig. 30. Comparison between the actual and identified cross-section area profiles.

In order to verify the suitability of the adopted techniques, distinct damage shapes and positions were considered, as well as different damage intensities. Furthermore, four different levels of SNR were provided in order to examine the actual capability of the methods to deal with noise corrupted data. In addition, a Dirac's delta impulse and two finite rectangular pulse excitations were considered.

To validate both the SAA direct method and the optimization techniques, a straightforward pulse-echo experiment was performed, for one damage profile. The experimental result showed that the synthetic echo reproduces pretty well the experimental one. Furthermore, the experimental damage profile showed to be satisfactorily recovered by the identification procedure.

One of the main advantages of the acoustic wave propagation approach is that it allows to identify, prior to the damage identification procedure itself, the damage location. In other words, analyzing directly the echoes, it is possible to infer about the damage location. For instance, inspecting the echoes plotted in Fig. 2, it is easy to find both the position and extension of the damage. Only its shape needs the optimization procedure.

The first conclusion is that both methods lead to excellent identification results in the absence of additive noise, as shown in Figs 4, 5, 7 and 8. Naturally, due to its stochastic nature, the PSO method presented a residual error in the identification results, while the PSO-LM method did not.

For the damage assessment based on the impulse response, the tests run with SNR of 30 and $20 \mathrm{~dB}$, i.e., with low and moderate noise levels, the performance was quite the same as that with noiseless data, for all considered damage cases, as can be seen in Figs 10a and 10b. For the tests run with SNR of 10 and 0 dB, i.e., with high and very high noise levels, the performance showed to be still good, with the PSO-LM method presenting slightly better identification results, for all tested damages. However, as shown in Tables 2 and 3, the adopted hybrid PSO-LM method presents a higher computational efficiency.

When considering rectangular pulse excitations and SNR of $10 \mathrm{~dB}$, the damage profile was recovered as well, as shown in Figs 23 and 24. The identification results were similar to that one based on the impulse response, as seen in Fig. 14a. One of the important conclusions is that the general formula for the echo, Eqs (5), (6), and (7) yield an identification procedure that is robust with respect to the input signal.

Observing the identification relative errors, as shown in Figs 16 to 20, one can conclude that, for a moderate SNR of $20 \mathrm{~dB}$, the maximum error for all methods and all damage cases is not greater than $0.8 \%$. As expected, the average error of the PSO method is greater than that of the PSO-LM. For the worst noise situation, i.e., SNR of $0 \mathrm{~dB}$, the maximum error found in all cases was lower than 6\%. Observing Figs 23 and 24, one can see that for SNR $=10 \mathrm{~dB}$ and rectangular pulse excitations, the relative errors stayed below 4\%, which are still satisfactory results.

It is worth noting that the damage identification procedure built on the SAA is robust with respect to noise corrupted signals, yielding to satisfactory results even in the presence of noise. The experimental results presented in Section 6 shows that the assumptions of the adopted models for the direct and inverse problems are realistic and in agreement to the actual dynamic behavior.

Finally, it should be mentioned that due to the SAA model shortly summarized in Subsection 2.1, the PSO method can be easily applied since only one parameter may be identified at once instead of all parameters together as should be done, for instance, when leading directly with the non-homogeneous hyperbolic wave equation. And, of course, this facilitates, as a natural consequence, the application of the hybrid PSO-LM technique. 


\section{Acknowledgments}

The authors acknowledge the Brazilian National Council for Scientific and Technological Development, CNPq, and Rio de Janeiro's Foundation for Research Support, FAPERJ, for their financial support to this research.

\section{References}

[1] J.V. Araújo dos Santos, N.M.M. Maia, C.M. Mota Soares and C.A. Mota Soares, Structural Damage Identification: A Survey, in: Trends in Computational Structures Technology, (Chapter 1), B.H.V. Topping and M. Papadrakakis, eds, Saxe-Coburg Publications, Stirlingshire, UK, 2008, pp. 1-24. doi:10.4203/csets.19.1.

[2] R. Arkin, Behavior-Based Robotics, MIT Press, 1998.

[3] J.C. Becceneri, S. Stephany, H.F.C. Velho and A.J. Silva Neto, Solution of the Inverse Problem of Radiative Properties Estimation with the Particle Swarm Optimization Technique, Proceedings of the Inverse Problems in Engineering Seminar, Ames, 2006.

[4] O. Begambre and J.E. Laier, A hybrid Particle Swarm Optimization - Simplex algorithm (PSOS) for structural damage identification, Advances in Engineering Software 40 (2009), 883-891.

[5] F. van den Bergh and A. Engelbrecht, Cooperative Learning in Neural Networks using Particle Swarm Optimizers, Proceedings of the SAICSIT, South Africa, 2000.

[6] E. Bonabeau, M. Dorigo and G. Theraulaz, Swarm Intelligence: From Natural to Artificial Systems, Oxford University Press, 1999.

[7] J.H. Cantrell and W.T. Yost, Nonlinear ultrasonic characterization of fatigue microstructures, Int J Fatigue 23 (2001), 487-480.

[8] K.M. Fernandes, L.T. Stutz, R.A. Tenenbaum and A.J. Silva Neto, Vibration and Wave Propagation Approaches Applied to Assess Damage Influence on the Behavior of Euler-Bernoulli Beams - Part II: Inverse Problem, Proceedings of the Ninth International Conference on Computational Structures Technology, Athens, Paper 39, 2008.

[9] R. Gangadharan, D. Roy Mahapatra, S. Gopalakrishnan, C.R.L. Murthy and M.R. Bhat, On the sensitivity of elastic waves due to structural damages: Time-frequency based indexing method, Journal of Sound and Vibration 320 (2009), 915-941.

[10] J. Grabowska, M. Palacz and M. Krawczuka, Damage identification by wavelet analysis, Mechanical Systems and Signal Processing 22 (2008), 1623-1635.

[11] Z. He, C. Wei, L. Yang, X. Gao, S. Yao, R. Eberhart and Y. Shi, Extracting Rules from Fuzzy Neural Network by Particle Swarm Optimization, Proceedings of the IEEE International Conference on Evolutionary Computation, Anchorage, 1998.

[12] J. Kennedy, The particle swarm: Social adaptation of knowledge. Proceedings of the 1997 International Conference on Evolutionary Computation, pp. 303-308, IEEE Service Center, Piscataway, 1997

[13] J. Kennedy, The behavior of particles. Proceedings of the 7th International Conference on Evolutionary Programming, pp. 581-587, San Diego, 1998.

[14] J. Kennedy and R.C. Eberhart, Swarm Intelligence. Morgan Kaufmann Publishers, 2001.

[15] J. Kennedy and R.C. Eberhart, Particle Swarm Optimization, Proceedings of the IEEE International Conference on Neural Networks, pp. 1942-1948, Piscataway, 1995.

[16] S. Küchler, T. Meurer, L.J. Jacobs and J. Qu, Two-dimensional wave propagation in an elastic half-space with quadratic nonlinearity: A numerical study, J Acoust Soc Am 125 (2009), 1293-1301.

[17] U. Lee, A reduced-domain method of structural damage identification: Application to a spectral element beam model, Shock and Vibration 10 (2003), 313-324.

[18] J.P. Lefebre, La tomographie d'impédance acoustique, Trait Signal 2(2) (1985), 103-110.

[19] W. Lestari, Application of wave propagation analysis for damage identification in composite laminated beams, J. Composite Materials 39(22) (2005), 1967-1984.

[20] G. Manson and R.J. Barthorpe, Advanced feature selection for simplified pattern recognition within the damage identification framework, Shock and Vibration 17 (2010), 589-599.

[21] D.W. Marquardt, An algorithm for least-squares estimation of nonlinear parameters, J Soc Industr Appl Math 11 (1963), $431-441$.

[22] J.M. Mendell and F.H. Ashrafi, A survey of approaches to solving inverse problems for lossless layered media systems, IEEE Trans Geosci Rem Sensing GE-18(4) (1980), 320-330.

[23] D. Montalvão, N.M.M. Maia and A.M.R.A. Ribeiro, Review of vibration-based structural health monitoring with special emphasis on composite materials, The Shock and Vibration Digest 38 (2006), 295-324.

[24] A. Nag, D.R. Mahapatra and S. Gopalakrihnan, Identification of delamination in composite beams using spectral estimation and a genetic algorithm, Smart Materials and Structures 11(6) (2002), 899-908.

[25] P.B. Nagy, Fatigue damage assessment by nonlinear ultrasonic material characterization, Ultrasonics 36 (1998), $375-381$.

[26] S.H. Ni, K.F. Lo, L. Lehmann and Y.H. Huang, Time-frequency analyses of pile-integrity testing using wavelet transform, Computers and Geotechnics 35 (2008), 600-607.

[27] S.H. Nia, J.J. Charngc and K.F. Lod, Low-strain integrity testing of drilled piles with high slenderness ratio, Computers and Geotechnics 33 (2006), 283-293.

[28] E. Niederleithinger and A. Taffe, Early stage elastic wave velocity of concrete piles, Cement and Concrete Composites 28(4) (2006), 317-320.

[29] A.D. Pierce, Acoustics: An Introduction to its Physical Principles and Applications, Acoustical Society of America, Woodbury, 1989.

[30] C. Pruell, J.Y. Kim, J. Qu and L.J. Jacobs, Evaluation of plasticity driven material damage using Lamb waves, Appl Phys Lett 91 (2007), 231911. 
[31] M.A. Rao, J. Srinivas and B.S.N. Murthy, A Damage detection in vibrating bodies using genetic algorithms, Computers and Structures 82 (2004), 963-968.

[32] E.A. Robinson and S. Treitel, Geophysical Signal Analysis, Englewood Cliffs, Prentice Hall, 1980.

[33] S. Rucevskis and M. Wesolowski, Identification of damage in a beam structure by using mode shape curvature squares, Shock and Vibration 17 (2010), 601-610.

[34] H. Schwetlick, Inverse methods in the reconstruction of acoustic impedance profiles, J Acoust Soc Am 73 (1983), 1179-1186.

[35] A. Sheta, Reliability growth modeling for software fault detection using particle swarm optimization. Proceedings of the 2006 IEEE Congress on Evolutionary Computation, Vancouver, 2006.

[36] A.J. Silva Neto and F.J.C.P. Soeiro, Estimation of phase function of anisotropic scattering with a combination of gradient based and stochastic global optimization method. Proceeding of the 5th World Congress Computational Mechanics, Viena, 2002.

[37] R.A. Tenenbaum and M. Zindeluk, Classical signal processing techniques applied to variable impedance identification by impulse testing. Proc. of the $15^{\text {th }}$ Internoise, v. 2, 1241-1246, 1986.

[38] R.A. Tenenbaum and M. Zindeluk, An exact solution for the one-dimensional elastic wave equation in layered media, $J$ Acoust Soc Am 92(6) (1992), 3364-3370.

[39] R.A. Tenenbaum and M. Zindeluk, A fast algorithm to solve the inverse scattering problem in layered media with arbitrary input, $J$ Acoust Soc Am 92(6) (1992), 3371-3378.

[40] R.A. Tenenbaum, L.T. Stutz and K.M. Fernandes, Comparison of vibration and wave propagation approaches applied to assess damage influence on the behavior of Euler-Bernoulli beams, Computers and Structures, 2009.

[41] S. Vanaverbeke and K.V.D. Abeele, Two-dimensional modeling of wave propagation in materials with hysteretic nonlinearity, $J$ Acoust Soc Am 122 (2007), 58-72.

[42] H.F.C. Velho, F.M. Ramos, E.S. Chalhoub, S. Stephany, J.C. Carvalho and F.L. Souza, Inverse problems in space science and technology. Proceedings of the 5th International Conference on Industrial and Applied Mathematics, Sidney, 2003.

[43] J.R. Wait, G.H. Park and C.R. Farrar, Integrated structural health assessment using piezoelectric active sensors, Shock and Vibration 12 (2005), 389-405.

[44] S. Zhongqing, Y. Lin and L. Ye, Guided Lamb waves for identification of damage in composite structures: A review, Journal of Sound and Vibration 295 (2006), 753-780. 

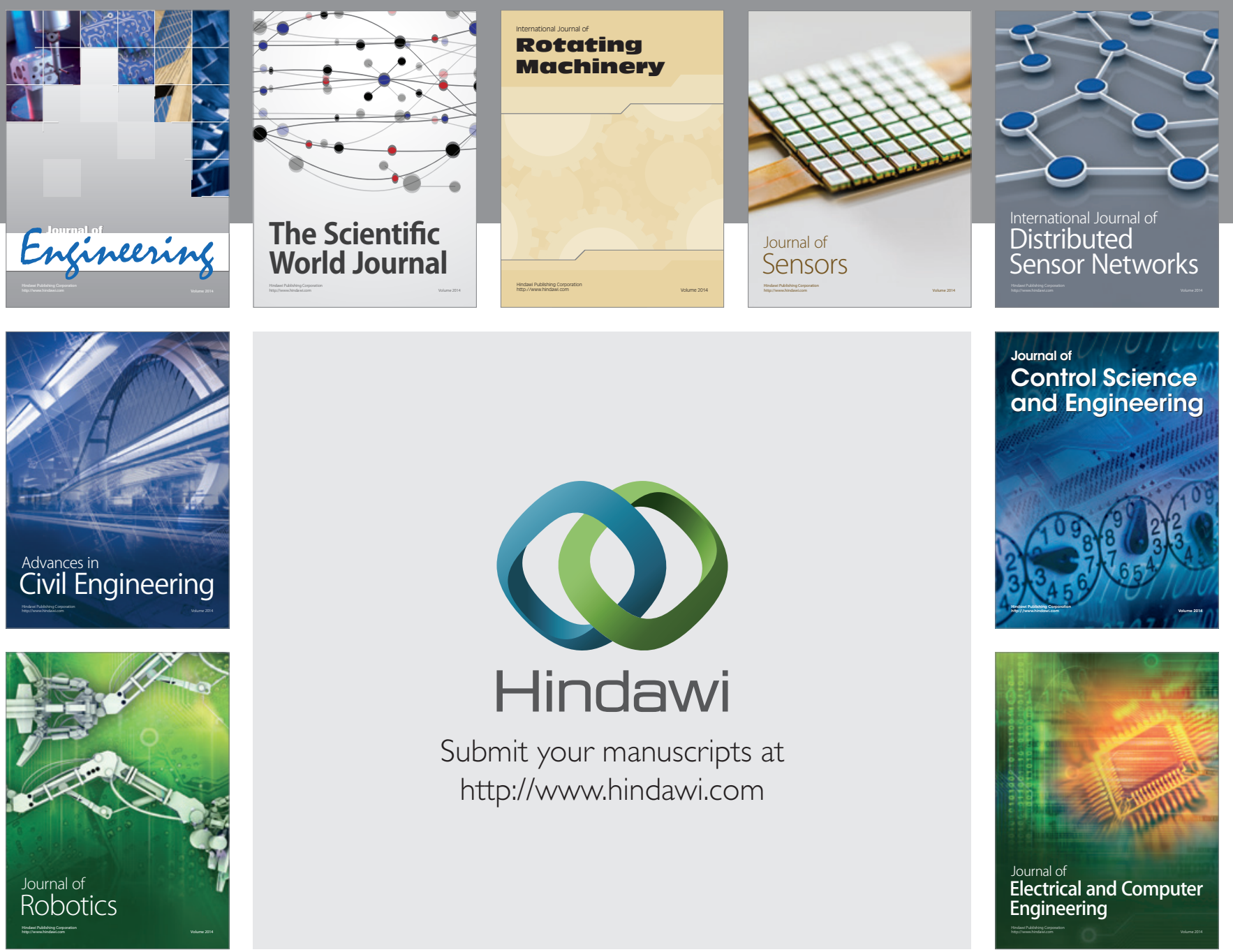

Submit your manuscripts at

http://www.hindawi.com
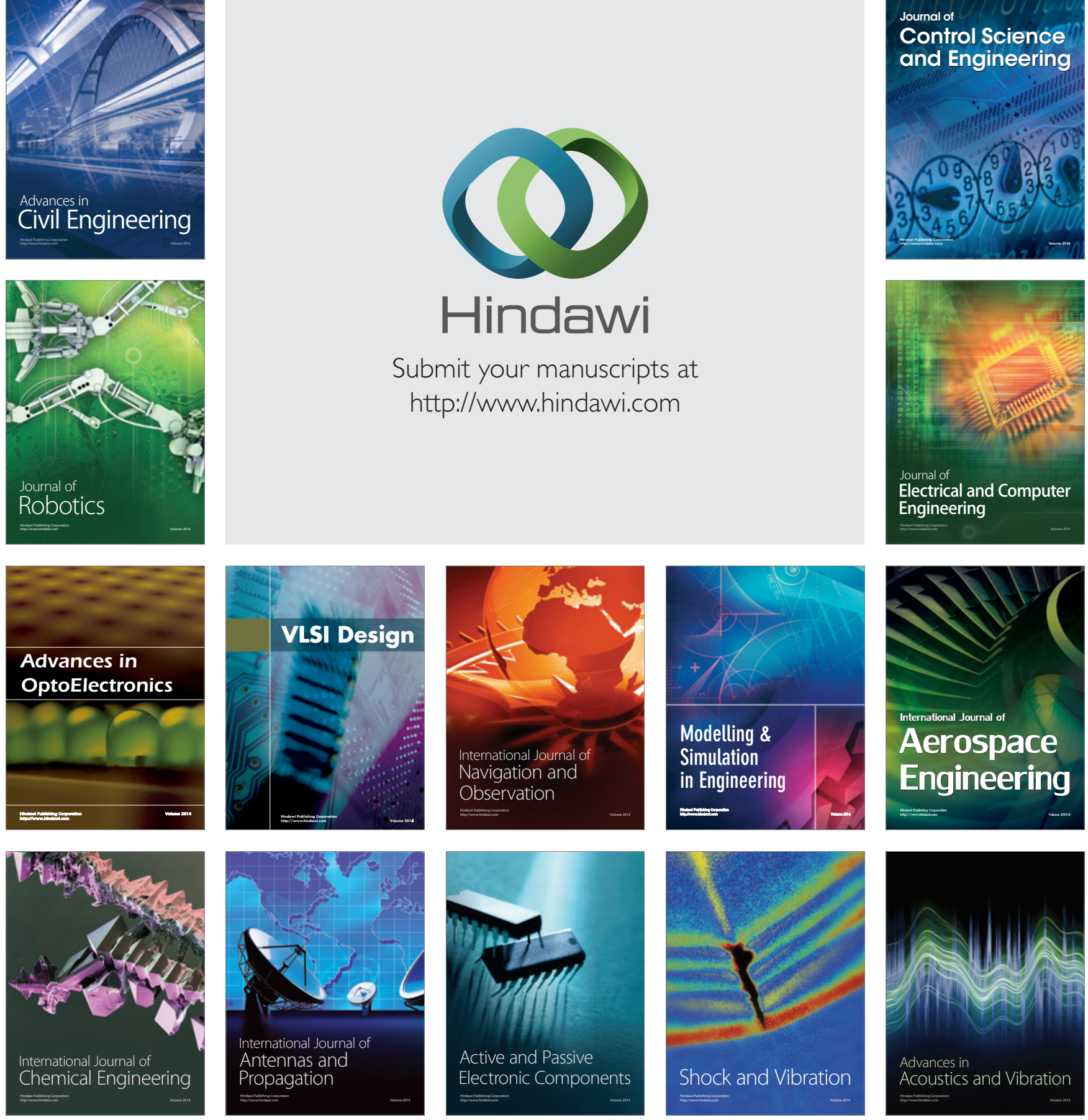